\title{
mTOR-regulated Mitochondrial Metabolism Limits Mycobacterium-induced
}

\section{Cytotoxicity}

Antonio J. Pagán ${ }^{1,2,3}$, Lauren J. Lee ${ }^{1,2}$, Joy Edwards-Hicks ${ }^{4,6}$, Cecilia B. Moens ${ }^{5}$, David

M. Tobin ${ }^{3,7}$, Elisabeth M. Busch-Nentwich ${ }^{1,8}$, Erika L. Pearce ${ }^{4,9}$, Lalita

Ramakrishnan ${ }^{1,2,3,10}$

${ }^{1}$ Molecular Immunity Unit, Cambridge Institute of Therapeutic Immunology and Infectious Diseases, Department of Medicine, University of Cambridge, Cambridge CB2 OAW, UK.

${ }^{2}$ MRC Laboratory of Molecular Biology, Cambridge CB2 OQH, UK

${ }^{3}$ Department of Microbiology, University of Washington, Seattle, WA 98195, USA

${ }^{4}$ Max Planck Institute of Immunobiology and Epigenetics, Freiburg im Breisgau, Germany.

${ }^{5}$ Division of Basic Sciences, Fred Hutchinson Cancer Center, Seattle, WA 98109, USA

${ }^{6}$ Present address: Cambridge Institute of Therapeutic Immunology and Infectious

Diseases, Department of Medicine, University of Cambridge, Cambridge CB2 OAW, UK

${ }^{7}$ Present address: Department of Molecular Genetics and Microbiology, Duke University, Durham, NC 27710, USA

${ }^{8}$ Present address: School of Biological and Behavioural Sciences, Faculty of Science and Engineering, Queen Mary University of London, London E1 4NS, UK.

${ }^{9}$ Present address: The Bloomberg-Kimmel Institute for Cancer Immunotherapy, Johns Hopkins University School of Medicine, Baltimore, Maryland, USA; Department of Oncology, Johns Hopkins University School of Medicine, Baltimore, MD 21287, USA.

${ }^{10}$ Lead contact

Correspondence: apagan@mrc-Imb.cam.ac.uk; lalitar@mrc-Imb.cam.ac.uk 


\section{ABSTRACT}

2 Necrosis of macrophages in the tuberculous granuloma represents a major pathogenic

3 event in tuberculosis. Through a zebrafish forward genetic screen, we identified the

4 mTOR kinase, a master regulator of metabolism, as an early host resistance factor in

5 tuberculosis. We found that mTOR complex 1 protects macrophages from

6 mycobacterium-induced death by enabling infection-induced increases in mitochondrial

7 energy metabolism fueled by glycolysis. These metabolic adaptations are required to

8 prevent mitochondrial damage and death caused specifically by ESAT-6, the principal

9 secreted substrate of the specialized mycobacterial secretion system ESX-1, a key

10 virulence mediator. Thus, the host can effectively counter this early critical

11 mycobacterial virulence mechanism simply by regulating energy metabolism, allowing

12 pathogen-specific immune mechanisms time to develop. Our findings may explain why

13 Mycobacterium tuberculosis, albeit humanity's most lethal pathogen, is successful in

14 only a minority of infected individuals. 


\section{INTRODUCTION}

17 Mycobacterium tuberculosis (Mtb) induces the formation of granulomas, organized structures comprised of macrophages, within which mycobacteria reside, and accessory

19 cells (Pagan and Ramakrishnan, 2014; 2018; Ramakrishnan, 2012). This hallmark

20 immunological structure of tuberculosis (TB) represents a key host-pathogen

21 battleground that determines the outcome of infection (Pagan and Ramakrishnan, 2014;

22 Ramakrishnan, 2012). In the majority of individuals, the granuloma is successful in

23 clearing Mtb infection, often leaving a residual, sterile, fibrotic structure as a stamp of

24 past infection (Behr et al., 2018; 2019; Behr et al., 2021; Canetti et al., 1972; Feldman

25 and Baggenstoss, 1938; Opie and Aronson, 1927; Terplan, 1951). In contrast, in the

26 minority of individuals who go on to develop TB, the granuloma is often rendered a

27 mycobacterium-beneficial structure that promotes bacterial expansion and

28 dissemination (Pagan and Ramakrishnan, 2014; Ramakrishnan, 2012). Granuloma

29 necrosis constitutes a pivotal pathogenic event because it delivers macrophage-resident

30 mycobacteria into the extracellular milieu, which increases their replication by

31 overcoming the growth restriction imposed by macrophage immune defenses (Pagan

32 and Ramakrishnan, 2014; Ramakrishnan, 2012; Russell, 2007). Necrosis of lung

33 granulomas with their attendant rupture into the airways also facilitates transmission,

34 sustaining the global TB burden and promoting Mtb's evolutionary survival (Ong et al.,

35 2014; Ramakrishnan, 2012). Exploitation of the granuloma begins in the innate stage of

36 the response, enabling Mtb to gain a foothold in the host (Cambier et al., 2014a; Pagan

37 and Ramakrishnan, 2014; Ramakrishnan, 2012). Innate immune defects that facilitate

38 mycobacterial exploitation of the granuloma, including accelerated granuloma necrosis, 
39 may hinder the development of host-protective adaptive immune responses.

40 Conversely, as adaptive immunity elaborates inflammatory programs set in motion by

41 innate immunity, it likely contributes to TB progression through the amplification of

42 pathogenic innate inflammatory responses (Pagan and Ramakrishnan, 2014; 2018).

43 Thus, the extent of mycobacterial exploitation of the granuloma - from its inception and

44 throughout the infection - may influence TB disease course.

45 Zebrafish infected with Mycobacterium marinum (Mm), a close relative of Mtb

46 develop a TB-like disease featuring the necrotic granulomas of human TB (Cosma et

47 al., 2003; Swaim et al., 2006). Mm-infected zebrafish larvae, in which adaptive immunity

48 has not yet developed, also develop disease with organized granulomas that undergo

49 necrosis (Davis et al., 2002). Thus, the zebrafish larva offers the opportunity to dissect

50 the sole contribution of innate immunity in tuberculous granuloma formation and

51 necrosis (Davis et al., 2002; Davis and Ramakrishnan, 2009; Ramakrishnan, 2013;

52 2020). The larva's optical transparency and genetic tractability enables the detailed,

53 sequential monitoring by intravital microscopy of the steps of infection, the contribution

54 of host and pathogen determinants to them, and the consequences to outcome

55 (Ramakrishnan, 2013). The use of unbiased genetic screens and candidate gene

56 approaches in zebrafish larvae has shed light on granuloma biology (Ramakrishnan,

57 2013; 2020). In particular, hypersusceptible zebrafish mutants displaying accelerated

58 granuloma necrosis have identified innate immune host determinants that protect

59 against necrosis and that are relevant in the context of human TB (Berg et al., 2016;

60 Clay et al., 2008; Pagan et al., 2015; Roca and Ramakrishnan, 2013; Roca et al., 2019;

61 Tobin et al., 2012; Tobin et al., 2010; Whitworth et al., 2021a; Whitworth et al., 2021b). 
Here, we report on insights into TB pathogenesis and resistance gained from

63 studies on a loss-of-function mutant in mTOR kinase that exhibits rapid granuloma

64 necrosis. The mTOR pathway integrates environmental signals emanating from diverse

65 nutrient-sensing and growth factor receptor pathways to regulate biosynthetic and

66 metabolic processes vital for cellular development, growth, survival, and function (Liu

67 and Sabatini, 2020). Iterative experimental approaches in the zebrafish and in human

68 macrophages uncover the mitotoxic function of mycobacterial ESAT-6 and show that

69 mTOR-facilitated mitochondrial metabolism serves as a highly effective innate 'counter

70 virulence' factor in TB by exerting a mitoprotective effect against this critical

71 mycobacterial virulence factor.

73 RESULTS

\section{4 mTORC1 Deficiency Confers Susceptibility to Mm Infection in Zebrafish}

75 The zebrafish mutant fh178, identified in a forward genetic screen (Tobin et al., 2010),

76 was hypersusceptible to $\mathrm{Mm}$, with larvae exhibiting increased bacterial burdens relative

77 to wild-type and heterozygote siblings following intravenous infection into the caudal

78 vein (CV) (Figures 1A-1C). By four days post infection (dpi), fh178 mutant granulomas

79 had depleted their macrophages, with the released bacteria growing exuberantly,

80 forming the rope-like cords that are characteristic of extracellular growth (Pagan et al.,

81 2015; Tobin et al., 2010) (Figure 1D and 1E). Mycobacterial cording, a sensitive and

82 specific surrogate for macrophage depletion, is a readily quantifiable, binary phenotype

83 for mapping mutants rendered hypersusceptible by granuloma necrosis (Berg et al.,

84 2016; Tobin et al., 2010). Using simple sequence length polymorphism markers, we 
85 thus mapped the fh178 mutation to a nonsense mutation in exon 24 of the mtor gene.

86 We confirmed that genetic disruption of mtor was the cause of fh178 hypersusceptibility

87 by showing that animals with a nonsense mutation in exon 19 (mtor $^{\text {sa16755 }}$ identified in

88 the Zebrafish Mutation Project (Kettleborough et al., 2013)) also exhibited

89 hypersusceptibility with cording as did compound fh178/sa16755 heterozygotes (Figure

$90 \quad 1 F$ and $1 G)$.

91 The mTOR kinase functions in two distinct molecular complexes, mTORC1 and

92 mTORC2, that require the adaptors Raptor and Rictor for their assembly, respectively

93 (Liu and Sabatini, 2020). Animals with nonsense alleles of rptor, the gene encoding

94 Raptor, showed similar cording as the mtor mutants, whereas those with nonsense

95 alleles of the zebrafish orthologs of mammalian Rictor, rictora and rictorb, did not

96 (Figure 1H and 1I). Pharmacological inhibition of mTOR or mTORC1 with torin1 or

97 rapamycin, respectively (Benjamin et al., 2011), recapitulated genetic mTOR/mTORC1

98 deficiency with increased cording (Figure 1J). Thus, mTORC1 deficiency confers

99 susceptibility to mycobacterial infection, linked to early granuloma breakdown. Because

100 zebrafish larvae have not yet developed adaptive immunity, this reflects resistance

101 conferred by mTOR in the sole context of innate immunity.

102

103 mTOR Deficiency Accelerates Death of Mycobacterium-Infected Macrophages

104 At 4 dpi, wild-type animals had granulomas with sparse intracellular bacteria; in contrast

105 mTOR-deficient animals had clusters of extracellular bacteria (Figure 1D). These

106 extracellular clusters had similar shapes to wild-type granulomas (Figure 1D, compare

107 top and bottom), suggesting that granulomas had formed initially and then broken down 
108 due to macrophage death. To detail the kinetics of macrophage death, we infected

109 zebrafish larvae in the hindbrain ventricle (HBV), an acellular compartment lined with a

110 single layer of epithelium that allows detailed monitoring of macrophage recruitment and

111 granuloma formation (Davis and Ramakrishnan, 2009) (Figure 1A). Rapamycin-treated

112 animals formed tight aggregates of infected macrophages similar to wild-type by 2 dpi

113 (Figure 2A). However, the next day, most macrophages in the nascent granulomas of

114 the rapamycin-treated animals had died, leaving a clump of extracellular bacteria

115 (Figure 2A).

A ready explanation for these findings could have been that, since mTORC1

117 signaling is required for myeloid cell development and maintenance, its deficiency would

118 create a basal macrophage deficit leading to susceptibility (Deng et al., 2017; Karmaus

119 et al., 2017; Lee et al., 2017; Mercalli et al., 2006; Sinclair et al., 2017; Weichhart et al.,

120 2015). We had previously identified susceptibility from a basal macrophage deficit. We

121 had found that zebrafish larvae deficient in the myeloid growth factor Colony Stimulating

122 Factor-1 Receptor (CSF-1R) are susceptible to Mm infection because they have a

123 reduced reservoir of uninfected macrophages available to replenish mycobacterium-

124 overladen macrophages dying in granulomas (Pagan et al., 2015). Further supporting

125 this possibility, mTORC1 promotes myelopoiesis at least in part through CSF-1R

126 signaling (Karmaus et al., 2017), and mTORC1 (rptor)-deficient zebrafish did have

127 reduced macrophage numbers in mock-infected animals (Figure 2B). However,

128 although mTORC1-deficient animals had more macrophages at baseline than CSF-1R-

129 deficient animals, their granulomas broke down at an earlier time-point: bacterial

130 cording occurred between days two and four in mTORC1-deficient animals versus 
131 between days five and seven in CSF-1R-deficient animals (Pagan et al., 2015)(Figure

132 2C). This suggested that mTOR deficiency induces death of infected granuloma

133 macrophages independently of reducing basal macrophage supply.

To show this definitively, we used time-lapse microscopy to capture in real-time

135 the death of infected wildtype and mTOR-deficient macrophages. If mTOR-deficient

136 granulomas are breaking down due to reduced macrophage replenishment, then dying

137 mTOR mutant macrophages should have bacterial burdens similar to or greater than

138 wild-type. This would also be the case if mTOR deficiency reduced the microbicidal

139 capacity of macrophages, so that dying macrophages would be overladen with bacteria.

140 To directly assess bacterial burdens in dying macrophages, we used blue fluorescent

141 Mm to infect mTOR-deficient animals and their wild-type siblings that had red

142 fluorescent macrophage membranes. All animals were also transgenic for a

143 ubiquitously-expressed secreted annexin $\mathrm{V}$ tagged with yellow fluorescent protein

144 (Tg(ubb:secA5-YFP)), which accumulates on the surface of cells undergoing apoptosis

145 and other modes of regulated cell death (Bendall and Green, 2014). We monitored

146 macrophage death by the appearance of annexin V-YFP labeling of the plasma

147 membrane and membrane blebs followed by the loss of tdTomato fluorescence

148 reflecting plasma membrane disintegration (Figure 2D and Movie S1). In 2 dpi animals,

149 over a 4.5-hour imaging period that captured similar numbers of infected macrophages

150 in mTOR-deficient and wildtype siblings, 6-fold more mTOR-deficient macrophages died

151 (Figure 2E and 2F and Movie S2). Importantly, mTOR-deficient macrophages died with

152 lower bacterial burdens than mTOR-sufficient macrophages than wild-type (Figure 2G), 
153 suggesting that they are "intolerant" of mycobacterial infection. These findings also ruled

154 out the possibility that mTOR deficiency causes a macrophage microbicidal defect.

155 Next, to determine if mTOR's protective effect was macrophage-intrinsic, we

156 compared mTOR mutant and wild-type macrophages in the same environment by

157 creating wildtype - mTOR mutant parabionts with differentially labeled macrophages,

158 red and yellow fluorescent in wild-type and mTOR mutants, respectively and infecting

159 both caudal veins (Figure $2 \mathrm{H}$ ). By 4 dpi, mTOR mutant macrophages had been

160 depleted by more than $90 \%$ on both wild-type and mTOR mutant sides of the

161 parabionts, whereas wild-type macrophages persisted equally on both sides (Figure 2l).

162 The depletion of mTOR mutant macrophages in these animals should not lead to Mm

163 cording because the complement of WT macrophages should be able to efferocytose

164 the infected mTOR mutant corpses and keep Mm intracellularly. By 4 dpi, as predicted,

165 Mm was only found inside the WT macrophages (Figure 2J). In mTOR heterozygote -

166 wildtype parabionts, mTOR heterozygote yellow fluorescent macrophages survived as

167 well as wild-type red fluorescent macrophages, ruling out expression of the

$168 \mathrm{Tg}$ (mpeg1:YFP) transgene as an artifactual cause of macrophage depletion of mTOR

169 mutant macrophages (Figure $2 \mathrm{~K}-2 \mathrm{~L}$ ).

170 Thus, mTOR confers cell-intrinsic protection to macrophages against

171 mycobacterium-induced death from early on even when intracellular bacteria are

172 sparse.

174 Infected macrophage death in mTOR deficiency is associated with reduced

175 membrane potential 
176 Impairment in nutrient sensing pathways caused by mTOR-deficiency could sensitize

177 cells to autophagic death or to mitochondrial apoptosis (Gonzalez et al., 2020; Green

178 and Levine, 2014; Hosoi et al., 1999; Muthukkumar et al., 1995). To assess autophagic

179 death, we created zebrafish deficient in the essential autophagy protein ATG12 (Bento

180 et al., 2016). Macrophages in ATG12-deficient animals had defective autophagosome

181 formation as evidenced by reduced aggregation of the autophagy protein LC3 (Figures

182 S1A and S1B). However, ATG12 deficiency did not prevent death of mycobacterium-

183 infected macrophages in animals rendered mTOR-deficient by rapamycin treatment

184 (Figure S1C). Next, to assess mitochondrial apoptosis, we used caspase-9 deficient

185 zebrafish mutants (Galluzzi et al., 2016). Capase-9 mutants had the expected defect in

186 developmental apoptosis as reflected by reduced dead cell debris in the brain at $3 \mathrm{dpf}$

187 (S1D and S1E). However, rapamycin treatment still induced death of their infected

188 macrophages (Figure S1F). Thus, mTOR deficiency kills infected macrophages

189 independent of inducing autophagy or apoptosis.

190 Mycobacterium-induced macrophage death typically occurs with high intracellular

191 bacterial burdens (Amaral et al., 2019; Beckwith et al., 2020; Clay et al., 2008; Lee et

192 al., 2011), whereas mTOR-deficient macrophages died rapidly upon infection when

193 intracellular mycobacterial burdens were still low. In previous work, we had identified

194 macrophage death associated with low mycobacterial burdens and found it to be

195 mediated through a pathway activated by dysregulated Tumor Necrosis Factor (TNF)

196 (Roca and Ramakrishnan, 2013; Roca et al., 2019). We found that mTOR-deficient

197 macrophage death did not involve this pathway; inhibition of essential components, the

198 kinase RIPK1 and the $L$ type calcium channels, failed to rescue death (Figure S1G - 
199 S1I). However, interrogating this pathway gave us a clue. TNF-mediated death of

200 mycobacterium-infected macrophages is initiated by high mitochondrial reactive oxygen

201 species (mROS) (Roca and Ramakrishnan, 2013; Roca et al., 2019), whereas in mTOR

202 deficient animals, both uninfected and infected macrophages had lower baseline mROS

203 levels than in wild-type (Figure 3A and B). Moreover, while mTOR-deficient

204 macrophages did mount the expected infection-induced increase in mROS, it was lower

205 than wild-type (1.6-fold versus 2.1-fold) (Figure 3B). Macrophage death associated with

206 low mROS levels implicated impaired mitochondrial function, which would be consistent

207 with mTOR's role in regulating mitochondrial biology (Cunningham et al., 2007; Liu and

208 Sabatini, 2020; Morita et al., 2013; Rambold and Pearce, 2018; Schieke et al., 2006).

209 To further explore the link between mTOR-deficiency, impaired mitochondrial

210 bioenergetics and death of infected macrophages, we rendered the THP-1 human

211 macrophage cell line mTOR-deficient by overnight treatment with the mTOR inhibitor

212 torin1 prior to infection. As with the zebrafish, there was increased death of infected

213 mTOR-deficient macrophages one day post-infection as shown by flow cytometry after

214 staining with the cell membrane-impermeant fixable viability dye eFluor 660 (Figure 3C

215 and 3D). Increases in mROS are linked to increased mitochondrial membrane potential

216 (Murphy, 2009), and accordingly, flow cytometry after tetramethylrhodamine, ethyl ester

217 (TMRE) staining showed that the infected wild-type macrophages had increased TMRE

218 (Figure 3E and 3F). As was the case with mROS levels, mTOR-deficient macrophages

219 had a lower baseline TMRE, which increased with infection but not to the extent in wild-

220 type infected cells (Figure 3E and F). 
Finally, death of infected mTOR signaling-deficient macrophages was associated

222 with mitochondrial damage - a greater proportion of the infected cells had released

223 cytochrome c (Figure 3G and $3 \mathrm{H}$ ). In contrast, there was hardly any cytochrome c

224 release in the uninfected cells (Figure 3G and $3 \mathrm{H}$ ). In sum, our findings suggest that

225 mycobacterium infection of mTOR-deficient zebrafish and human macrophages rapidly

226 induces a mitochondrial mode of death that is associated with reduced mitochondrial

227 metabolic activity.

229 mTOR Deficiency Sensitizes Macrophages to Mycobacterium-Induced Death by

230 Impairing Glycolysis-dependent Oxidative Phosphorylation (OXPHOS)

231 mTOR regulates a number of metabolic pathways that, in turn, regulate mitochondrial

232 metabolism (Liu and Sabatini, 2020). We confirmed that mTOR inhibition of THP-1 cells

233 caused the expected reduced activity in glucose metabolism (glycolysis and the pentose

234 phosphate pathway) and the Krebs cycle (Duvel et al., 2010; Morita et al., 2013),

235 reflected by lower metabolite abundance for these pathways consistent with reduced

236 glycolytic and respiratory capacity (Figure S2A - S2G). The lower basal, ATP-coupled

237 and maximal respiration, and spare respiratory capacity reflected broadly compromised

238 mitochondrial respiration and ATP production and reduced ability to boost mitochondrial

239 respiration in response to increased ATP demands. Together, these findings suggested

240 that mTOR deficiency impairs mitochondrial respiration by reducing glycolysis.

241 Consistent with this, selective inhibition of glycolysis with the glucose analog 2-deoxy-D-

242 glucose (2DG), which inhibits hexokinase and glucose-6-phosphate isomerase function

243 upon intracellular conversion into 2-deoxy-D-glucose-6-phosphate (Pajak et al., 2019) 
244 reduced Krebs cycle metabolites and mitochondrial respiration (Figure S2A, S2H, and

245 S2l and Table S1). Moreover, glycolysis-deficient macrophages had similar reductions

246 in ATP to mTOR-deficient ones (Figure S2J).

247 Our findings were consistent with a model where mTOR-facilitated glycolytic

248 fueling of the Krebs cycle sustains mitochondrial energy production required to protect

249 infected macrophages from dying. If so, then inhibition of glycolysis should phenocopy

250 mTOR deficiency, causing selective death of infected macrophages with impaired

251 infection-induced increase in mitochondrial membrane potential. 2DG treatment induced

252 both phenotypes in THP1 cells, similar to Torin1 treatment (Figure 4A and 4B). We

253 confirmed that mTOR and glycolysis have a cytoprotective effect in Mtb infection as

254 well. For this, we assessed macrophage death in after infection with an isogenic leucine

255 and pantothenate auxotrophic mutant of the virulent H37Rv Mtb strain, a biosafety 2

256 level pathogen that elicits similar inflammatory responses and triggers diverse cell death

257 programs (Beckwith et al., 2020; Mouton et al., 2019; Roca et al., 2019; Sampson et al.,

258 2004; Sampson et al., 2011). Mtb infection caused increased death of both mTOR-

259 deficient and glycolysis-deficient THP-1 cells (Figure 4C). Finally, in the zebrafish we

260 found that 2DG treatment depleted infected macrophages selectively (Figure 4D-4F and

261 Movie S3), and that this was associated with increased bacterial cording (Figure 4G).

262 Thus, our findings suggest that mTOR exerts its cytoprotective effect by supporting

263 glycolysis both in Mm zebrafish infection and Mm- and Mtb-infected human

264 macrophages.

265 Since glycolysis contributes to mitochondrial ATP production by supplying

266 pyruvate to the Krebs cycle (Ryan and O'Neill, 2020), intercepting this step should also 
267 produce bacterial cording. To test this, we treated zebrafish with the mitochondrial

268 pyruvate carrier inhibitor UK5099 (Halestrap, 1975). UK5099 treatment increased

269 bacterial cording (Figure 4H). Glycolysis also feeds the pentose phosphate pathway

270 (Patra and Hay, 2014), and we had observed alterations in pentose phosphate pathway

271 metabolites after inhibition of mTOR and glycolysis (Figure S2A). However, zebrafish

272 mutants deficient in glucose-6-phosphate dehydrogenase (G6PD), the rate-limiting

273 enzyme of the pentose phosphate pathway, which had 90\% reduction in G6PD activity,

274 did not have increased bacterial cording (Figure $4 \mathrm{I}$ and $4 \mathrm{~J}$ ). This ruled out the

275 involvement of the pentose phosphate pathway in mTOR's protective effect. Together,

276 these results suggest that mTOR deficiency sensitizes macrophages to Mm-induced

277 mitochondrial damage and death by impairing glycolysis-dependent OXPHOS and

278 thereby mitochondrial energy production. Consistent with this model, we found that

279 infection boosted ATP production within 24 hours in control macrophages (Figure 4K).

280 In contrast, mTOR-deficient macrophages had slightly lower baseline ATP production,

281 with infection increasing it only up to baseline wild-type levels (Figure 4K). This finding

282 recapitulates the findings with mROS and TMRE where mTOR-deficient macrophages

283 are able to mount a response to infection that equals or exceed wild-type baseline

284 levels but falls short of wild-type responses upon infection. Together, these findings

285 suggest that the increases in mitochondrial energy production in infected macrophages

286 are necessary to protect against mycobacterium-mediated mitochondrial damage.

287 mTOR protects against macrophage mitochondrial damage and death by enabling

288 these infection-induced metabolic adaptations. 
290 mTOR-dependent Glycolysis and OXPHOS Enable Macrophages to Resist

291 Mycobacterium ESAT-6-mediated Death

292 The mTOR mutant had unmasked the potential of pathogenic mycobacteria to cause

293 lethal mitochondrial damage in infected macrophages. We hypothesized that a specific

294 mycobacterial determinant caused this damage, and our prime candidate was the ESX-

2951 secretion system. ESX-1 is a major mycobacterial virulence determinant which, even

296 in normal (mTOR-sufficient) conditions, is implicated in mycobacterium-induced

297 macrophage death and mitochondrial damage (Conrad et al., 2017; Davis and

298 Ramakrishnan, 2009; Groschel et al., 2016; Guinn et al., 2004; Lienard et al., 2020;

299 Simeone et al., 2012; Simeone et al., 2021; Volkman et al., 2004; Wiens and Ernst,

300 2016). While ESX-1 does promote pathogenic death of mTOR-sufficient macrophages,

301 it is much more limited and occurs only when high intramacrophage bacterial burdens

302 are reached (Davis and Ramakrishnan, 2009; Volkman et al., 2004). We hypothesized

303 that mTOR-deficiency sensitizes macrophages to ESX-1-mediated mitochondrial

304 damage and cell death. Consistent with our hypothesis, mTOR-deficient THP-1

305 macrophages infected with ESX-1-deficient Mm did not have mitochondrial damage nor

306 increased death (Figure 5A and 5B). Furthermore, in mTOR-deficient zebrafish infected

307 with ESX-1-deficient Mm, time-lapse microscopy showed that infected macrophages did

308 not die (Figure 5C and Supplementary Movie 4). Accordingly, the animals did not exhibit

309 bacterial cording (Figure 5D). Finally, ESX-1-deficient infection did not cause

310 macrophage death in 2DG-treated and UK5099-treated animals either (Figure 5E and

$3115 F)$. Thus, mTOR-dependent glycolysis and OXPHOS specifically counter ESX-1-

312 dependent mitochondrial damage and cell death. 
ESX-1-mediated damage of mycobacterium-containing phagosomes is thought

314 to be upstream of its other virulence-related phenotypes such as macrophage death

315 and mitochondrial damage (Lienard et al., 2020; Simeone et al., 2012; Simeone et al.,

316 2021). The mycobacterial cell surface lipid phthiocerol dimycocerosate (PDIM)

317 facilitates ESX-1-mediated phagosomal damage and cytotoxicity (Augenstreich et al.,

318 2017; Lerner et al., 2018; Osman et al., 2020; Quigley et al., 2017; Simeone et al.,

319 2021) (Figure S3). We found that mTOR-deficient macrophages were similarly resistant

320 to mycobacteria deficient in the PDIM transporter MmpL7 (Cambier et al., 2014b) -

$321 \mathrm{mmpL7}$ mutants did not induce cording in mTOR mutants (Figure 5G). We further tested

322 mycobacterial mutants for the virulence determinants Erp and the serine protease

323 MarP, virulence determinants that promote intramacrophage growth through distinct

324 mechanisms from ESX-1(Berthet et al., 1998; Cosma et al., 2006; Ehrt et al., 2015;

325 Levitte et al., 2016; Small et al., 2013; Vandal et al., 2008). We confirmed that in wild-

326 type animals, the Erp and MarP mutants induced significantly more phagosomal

327 damage than ESX-1 and mmpL7 mutants, as determined by staining with an antibody to

328 galectin-8, a cytosolic protein that binds to lumenal $\beta$-galactoside-containing glycans

329 that become exposed on damaged vesicles (Figure S3) (Osman et al., 2020; Randow

330 and Youle, 2014). Despite their intramacrophage growth attenuation (Cosma et al.,

331 2006; Levitte et al., 2016), these mutants caused accelerated macrophage death in

332 mTOR-deficient animals (Figure 5G). These findings implicate ESX-1-dependent

333 phagosomal permeabilization in the accelerated death of mTOR-deficient macrophages.

334 ESX-1's membranolytic activity had been ascribed to its major secreted substrate

335 ESAT-6 (6 kDa early secretory antigenic target); however, pinning down its role versus 
336 those of other ESX-1 substrates has been complicated by their co-dependency for

337 secretion, as deletion of ESAT-6 causes loss of other ESX-1 substrates (Bao et al.,

338 2021; Champion et al., 2014; Fortune et al., 2005). Recent work has pinpointed a

339 specific role for ESAT-6 in phagosomal damage and virulence, by identifying point

340 mutations in ESAT-6 that allow substantial levels of secretion of ESAT- 6 and other

341 ESX-1 substrates yet cause loss of phagosomal membrane damage and/or virulence

342 (Brodin et al., 2005; Osman et al., 2022; Zhang et al., 2016). To test the specific role of

343 ESAT-6-induced phagosomal damage in mediating cell death in mTOR deficiency, we

344 used esxA mutant Mm expressing either of two such ESAT-6 C-terminal point

345 mutations, M83I and M93T (Brodin et al., 2005; Osman et al., 2022). Mm-ESAT-6 ${ }^{\text {M83I }}$

346 and Mm-ESAT- $6^{\mathrm{M} 93 \mathrm{~T}}$ infections did not kill macrophages in mTOR-deficient zebrafish

347 (Figure $5 \mathrm{H}$ ). These findings bolster the conclusion that mycobacterium-induced

348 phagosomal damage is an absolute requirement for the cell death induced by mTOR

349 deficiency and specifically implicate the ESX-1 substrate ESAT-6.

351 mTOR Enables Infected Macrophages to specifically resist ESX-1-mediated

352 mitochondrial damage

353 We considered the possibility that mTOR-deficiency simply sensitizes macrophage

354 phagosomes to ESX-1-mediated damage. However, mTOR-deficient and wildtype

355 macrophages had similar ESX-1-dependent increases in phagosomal damage, showing

356 that this was not the case and suggesting that mTOR-deficient macrophages were

357 sensitized to ESX-1-mediated mitochondrial rather than phagosomal damage (Figure

$3586 \mathrm{~A}$ and $6 \mathrm{~B})$. 
ESX-1-mitochondrial damage in mTOR-deficient macrophages could be indirect

360 or direct. In the indirect case, ESX-1 would permeabilize the phagosome allowing either

361 host lysosomal factors or a different mycobacterial determinant to damage the

362 mitochondria. In the direct case, ESX-1 would first damage phagosomes, which would

363 allow it to contact mitochondria and directly damage them. To distinguish between the

364 two, we treated THP-1 cells with prazosin, a drug that permeabilizes endo-lysosomal

365 compartments in myeloid cells (Kozik et al., 2020). By 7 hours of treatment, prazosin

366 had caused extensive endo-lysosomal damage in uninfected control and mTOR-

367 deficient macrophages as evidenced by galectin-8 staining (Figure S4). In infected

368 macrophages, prazosin caused similar increases in damaged wild-type and ESX-1-

369 deficient mycobacterial phagosomes (Figure 6B). Moreover, the proportion of wildtype

370 and ESX-1-mutant mycobacteria associated with damaged phagosomes was similar,

371 showing that the prazosin-induced phagosomal permeabilization could equalize access

372 of wildtype and ESX-1-deficient mycobacteria to the mitochondria (Figure 6C).

373 Consistent with increasing bacterial exposure to mitochondria, prazosin increased

374 cytochrome c release from wildtype-infected macrophages (Figure 6D). Prazosin-

375 treated ESX-1 mutant-infected macrophages had a much smaller increase in

376 cytochrome c release (33\% of prazosin-treated wild-type), and significantly lower than in

377 untreated wild-type macrophages (Figure 6D). These findings implicate ESX-1 in

378 directly mediating mitochondrial damage after first damaging the phagosome to enable

379 contact. The small increase in cytochrome c release in ESX-1-mutant infection suggest

380 that additional mechanisms may also play a role, for instance PDIM. To corroborate

381 these conclusions in vivo, we assessed bacterial cording in zebrafish with and without 
382 prazosin treatment. As before, mTOR deficiency caused cording of wildtype but not

383 ESX-1-deficient bacteria (Figure 6E). Prazosin treatment of mTOR mutants increased

384 ESX-1-deficient cording only slightly, and significantly less than wildtype bacteria

385 without prazosin (Figure 6E). These results show that ESX-1 possesses both

386 phagosomal and mitochondrial damaging activity and that mTOR protects specifically

387 against the latter. Our findings highlight the strength of mTOR as a "counter-virulence"

388 factor against ESAT- 6 and indicate that a small amount of ESAT-6 is sufficient to induce

389 cytotoxicity in mTOR-deficient macrophages - even small numbers of severely growth

390 - attenuated but ESX-1-competent mycobacteria rapidly induced cytotoxicity in them.

391 Furthermore, mTOR-deficient macrophages tolerated infection with mycobacteria if their

392 ESX-1 function was impaired. Furthermore, mTOR-deficient macrophages tolerated

393 infection with mycobacteria so long as they did not express one specific virulence

394 determinant. 


\section{DISCUSSION}

396 The association of infections, including TB, with therapeutic mTOR blockade in organ

397 transplantation and cancer is well recognized (Fijalkowska-Morawska et al., 2011;

398 Garcia and Wu, 2016; Jeon et al., 2017; Ruiz-Camps and Aguilar-Company, 2021; Tsai

399 et al., 2007). Since mTOR signaling in phagocytes, antigen-presenting cells, and T cells

400 is critical for their development, homeostasis and/or function, it is likely that mTOR

401 exerts its protective effects in TB through a myriad of mechanisms impacting innate and

402 adaptive immune responses (Lachmandas et al., 2016; Powell et al., 2012; Sinclair et

403 al., 2017; Weichhart et al., 2015). Our screen, undertaken in the sole context of innate

404 immunity, uncovers an essential early protective role for mTOR well before adaptive

405 immunity is generated. Still, our finding that mTOR confers innate resistance by

406 enhancing macrophage survival would have been expected given that mTOR is known

407 to support macrophage development and homeostasis and our previous findings that

408 sustained myelopoiesis is critically important to maintain granuloma cellularity (Deng et

409 al., 2017; Karmaus et al., 2017; Lee et al., 2017; Mercalli et al., 2006; Pagan et al.,

410 2015; Sinclair et al., 2017; Weichhart et al., 2015). The surprise is that mTOR deficiency

411 results in the rapid depletion of infected macrophages through catastrophic

412 mitochondrial damage caused specifically by ESAT-6/ESX-1, a major mycobacterial

413 virulence determinant. A corollary of this finding is that mitochondrial metabolism

414 represents a formidable armor against a potent mycobacterial mitotoxin. It is being

415 increasingly appreciated that while intracellular microbes can exploit host cell

416 metabolism for growth and pathogenesis, cellular metabolism, can in turn, use its 
417 metabolic capabilities to avert microbial attack (Pernas, 2021). Our findings reveal the

418 role of host metabolism in protection from a major infectious killer.

419 A major virulence attribute of ESX-1 is its ability to induce macrophage death

420 even in wild-type (mTOR competent) macrophages (Groschel et al., 2016;

421 Ramakrishnan, 2012). Studies in cultured cells have attributed this death to apoptosis,

422 pyroptosis or necrosis (Augenstreich et al., 2017; Beckwith et al., 2020; Behar et al.,

423 2010; Srinivasan et al., 2014) and the one in vivo study, also in zebrafish larvae, finds

424 that ESX-1 induces apoptosis of granuloma macrophages (Davis and Ramakrishnan,

425 2009). For all these forms of death, phagosomal damage is widely considered to be a

426 pre-requisite step, which is now confirmed to be mediated by ESAT-6 (Bao et al., 2021;

427 Osman et al., 2022; Simeone et al., 2021; Srinivasan et al., 2014; Zhang et al., 2016).

428 We show here that ESAT-6's ability to induce phagosomal damage is irrespective of

429 whether the macrophage is mTOR-sufficient or -deficient, a finding that is important for

430 its role in disease pathogenesis in normal hosts. Rather, we find that mTOR's specific

431 role is in resistance against ESX-1-mediated mitochondrial damage. While Mtb can also

432 damage the plasma membranes and mitochondrial membranes of mTOR-sufficient cells

433 once it has accessed them through ESX-1-mediated phagosomal damage, the factors

434 involved in those other forms of membrane damage remain unclear — studies have

435 variably implicated ESAT-6, other ESX-1 substrates, non-ESX-1 mycobacterial

436 products, excess lipoxins, and mROS (Beckwith et al., 2020; Chen et al., 2008;

437 Divangahi et al., 2009; Fine-Coulson et al., 2015; Lienard et al., 2020; Sun et al., 2015;

438 Wiens and Ernst, 2016). Here we show that ESX-1 directly induces mitochondrial

439 damage in mTOR-deficient cells. However, we have not yet shown whether the 
440 mitochondrial damage is through ESAT-6 or some other ESX-1 substrate. Similarly, we

441 and others have shown that PDIM facilitates ESAT-6 mediated phagosomal damage,

442 possibly by changing the characteristics of the mycobacterial surface, host membranes

443 or both (Augenstreich et al., 2017; Augenstreich et al., 2019; Cambier et al., 2020;

444 Osman et al., 2020; Simeone et al., 2021). In this work, we show that PDIM is essential

445 for the mitochondrial damage and death in mTOR deficiency but whether this effect is

446 solely by causing phagosomal damage or whether it additionally works with ESX-

447 1/ESAT-6 to damage mitochondrial membranes remains a question.

448 How does ESX-1 damage mitochondria? Mm and Mtb phagosomes frequently

449 fuse to lysosomes, and contacts between lysosomes and mitochondria are proposed to

450 facilitate metabolic exchanges between these organelles (Armstrong and Hart, 1971;

451 Barker et al., 1997; Clemens and Horwitz, 1995; Harris et al., 2008; Levitte et al., 2016;

452 Wong et al., 2018). The idea that mycobacterial phagolysosomes influence

453 mitochondrial function is reinforced by the finding that mitochondria aggregate around

454 these structures and undergo morphological changes (Jamwal et al., 2013; Mohareer et

455 al., 2020). Given that direct contact is required for ESX-1 permeabilization of host

456 membranes (Conrad et al., 2017), we speculate that ESAT-6 or a different secreted

457 ESX-1 substrate might specifically damage mitochondria that are in direct contact with

458 permeabilized phagosomes through sequential disruption of the outer and inner

459 mitochondrial membranes (Figure 7). Even at low levels of infection, damage to the

460 already metabolically-impaired mitochondria of mTOR-deficient macrophages would

461 further atrophy mitochondrial energetic metabolism, thereby promoting lethal reduction

462 in cellular energy reserves. This additional drop in energetic output would be expected 
463 to further impair the cell's ability to repair or replace damaged organelles, including

464 mitochondria.

465 Our findings provide insight into the adaptive metabolic changes that occur upon

466 mycobacterial infection. Stimulation of cultured macrophages with bacterial Toll-like

467 receptor (TLR) ligands like lipopolysaccharide (LPS) under normoxic conditions induces

468 a metabolic switch from mitochondrial OXPHOS to glycolysis through activation of

469 mTORC1 (O'Neill and Pearce, 2016; Weichhart et al., 2015). This switch enables

470 macrophages to elaborate antimicrobial responses that depend on cataplerosis of Krebs

471 cycle intermediates (Ryan and O'Neill, 2020). However, it is not clear to what extent

472 Mtb-infected macrophages undergo this glycolytic switch. Some studies find that Mtb

473 infection boosts glycolysis while others find that it represses it (Braverman et al., 2016;

474 Cumming et al., 2018; Gleeson et al., 2016; Huang et al., 2018; Lachmandas et al.,

475 2016; Shi et al., 2015). These conflicting findings may reflect the muted metabolic

476 response to mycobacterial infection in comparison to LPS, perhaps because they

477 largely evade TLR recognition (Cambier et al., 2014a; Cambier et al., 2014b; Hinman et

478 al., 2021; Holscher et al., 2008; Mayer-Barber et al., 2010). Indeed, in vivo, we have

479 shown that both Mm- and Mtb-infected macrophages exhibit small increases in

480 mitochondrial respiration early on (Roca, 2022). Our current work shows that these and

481 the resultant increases in ATP, which require mTORC1 signaling, though small,

482 constitute critical metabolic adaptations to cope with mycobacterial invasion.

483 Importantly, these metabolic adaptations are required only to resist ESX-1-induced

484 mitochondrial damage - mycobacteria not expressing ESX-1 are well tolerated by

485 macrophages lacking mTOR. 
While we cannot rule out adaptive glycolytic shifts later in infection, our data are consistent with mTORC1's role in resistance immediately upon mycobacterial invasion stemming from the boost in OXPHOS that comes from its stimulation of glycolysis (Weichhart et al., 2015). Indeed, our finding that inhibition of pyruvate import into mitochondria confers susceptibility highlights that glycolysis alone cannot compensate

491 for the deficits in mitochondrial metabolism caused by mTOR deficiency. mTOR

492 regulated biosynthetic processes may rapidly replace or repair damaged mitochondria,

493 consistent with the known roles of mTORC1 signaling mitochondrial physiology

494 (Cunningham et al., 2007; Liu and Sabatini, 2020; Morita et al., 2013; Morita et al., 495 2017; Rambold and Pearce, 2018; Schieke et al., 2006). Alternatively, these same 496 mTOR functions may ensure that there is a sufficient pool of functional mitochondria to 497 maintain the cell's energetic demands even when some mitochondria are damaged by 498 ESX-1. Furthermore, as macrophages need OXPHOS to activate antigen-specific T 499 cells and to respond to interferon- $\gamma$ (Kiritsy et al., 2021), maintaining mitochondrial

500 function in Mtb-infected macrophages may be a prerequisite for effective T cell501 mediated immunity in the granuloma. Induction of macrophage death is a major pathogenic mechanism used by

503 mycobacteria. Early on, apoptotic death of infected macrophages can promote

504 intercellular mycobacterial spread and thereby expansion of their numbers in early

505 granulomas (Davis et al., 2002; Ramakrishnan, 2012). ESX-1/ESAT-6 are implicated in

506 this apoptotic death as well as other forms of programmed cell death like pyroptosis and 507 necrosis, which involve plasma membrane damage (Augenstreich et al., 2017; Beckwith 508 et al., 2020; Behar et al., 2010; Divangahi et al., 2009; Srinivasan et al., 2014). ESX-1 is 
509 also implicated in the type-1 interferon (T1-IFN)-mediated cytotoxicity induced by Mtb

510 (Manzanillo et al., 2012; Mayer-Barber et al., 2014; Moreira-Teixeira et al., 2018;

511 Stanley et al., 2007; Zhang et al., 2021). While this form of death does involve

512 mitochondria - ESX-1-promoted mitochondrial DNA release stimulates cytosolic DNA-

513 sensing pathways (Hopfner and Hornung, 2020; Lienard et al., 2020; Wiens and Ernst,

514 2016) - its effects on the metabolism of both Mtb-infected and uninfected (bystander)

515 macrophages suggest a distinct cytotoxicity mechanism. T1-IFNs produced in response

516 to M. tuberculosis infection repress glycolysis and OXPHOS in both infected and

517 uninfected (bystander) macrophages and induce pathogenic levels of mROS in the

518 infected cells (Cumming et al., 2018; Olson et al., 2021; Wiens and Ernst, 2016). We

519 show that if mTOR is lacking, the above modes of ESX-1-mediated death become

520 irrelevant, because the cells die more rapidly from the inability to boost mitochondrial

521 metabolism in response to infection. Thus, the other forms of ESX-1-mediated death -

522 ultimately also mediated by its membranolytic activity — represent "workarounds" in

523 mycobacterium's pathogenic strategy.

$524 \quad$ TB is notorious for killing far more people over the millenia than any other

525 infectious agent (Paulson, 2013). Yet, our recent epidemiological analyses show that

526 most individuals clear Mtb infection through a combination of innate and adaptive

527 immunity (Behr et al., 2018; 2019; Behr et al., 2021). Understanding the immune

528 correlates of protection in the many is an active focus of TB researchers. Our work finds

529 that tapping into mTOR-regulated homeostatic metabolic pathways constitutes a major

530 host defense strategy. Curiously, these pathways protect against a specific

531 mycobacterial virulence determinant. Indeed, identifying mTOR as a "counter-virulence" 
532 factor against mycobacterium ESX-1 exemplifies TB as a paradigm of the host-

533 pathogen interaction, where the evolutionary survival of both host and pathogen are

534 ensured (Cambier et al., 2014a). mTOR buys the host time to call in other defenses that

535 can clear infection much of the time, while the success of the ESX-1 virulence

536 mechanism in the minority of infected individuals has assured Mtb survival over the

537 millennia.

\section{ACKNOWLEDGEMENTS}

540 We thank M. Behr, P. Edelstein, M. Murphy and F. Roca for discussions; M. Eisenberg-

541 Bord and N. Yamaguchi for manuscript review; K. Takaki for parabiosis instruction; C.

542 Hale for providing access to Seahorse XFp analyzer and advice on experimental

543 design; J. Ray for assistance mapping mtor ${ }^{\text {fh178; }}$ R. Berg, L. Hernández, S. Levitte, and

544 J. Zimmermann for assistance with early characterization of mtor $^{\text {fh178. }}$ H. Boyd, B.

545 Brockley, C. Dooley, S. McGuinan, R. Santhakumar, I. Smallwood, and R. White for

546 technical and logistical assistance with genetic screens; J. Cameron, N. Goodwin, R.

547 Foster, and the University of Cambridge aquatics facility staff for zebrafish husbandry;

548 the LMB's flow cytometry core facility and the NIHR Cambridge BRC Cell Phenotyping

549 Hub for equipment access, and the LMB's media service for preparation of bacterial and

550 tissue culture reagents. This work was supported by the National Institutes of Health

551 (T32-AI055396, A.J.P. and MERIT award R37-AI054503 and Director's Pioneer Award

552 DP1MH099901, L.R.), a Wellcome Trust Principal Research Fellowship (223103/Z/21/Z;

553 L.R.), the Max Planck Society (E.L.P.), and the Liebnietz Prize (E.L.P). 


\section{AUTHOR CONTRIBUTIONS}

557 A.J.P., C.B.M., D.M.T., and L.R. conceived the project. A.J.P., L.J.L., J.E.-H., C.B.M.,

558 D.M.T., E.M.B.-N., E.L.P. and L.R. designed experiments and analyzed data. A.J.P. and

559 L.J.L. performed zebrafish experiments. D.M.T. isolated and mapped $m t^{\text {th }}{ }^{\text {178 }}$. A.J.P.

560 conducted cell culture experiments. J.E.-H. performed and analyzed LC/MS. C.B.M. and

561 E.M.B.-N. provided chemically-mutagenized zebrafish lines. E.L.P. suggested and

562 supervised metabolomics experiments and advised on the experimental design and

563 interpretation of metabolic experiments. A.J.P. and L.R. wrote the manuscript and got

564 input from the other authors.

566 DECLARATION OF INTERESTS

568 E.L.P. is a scientific advisory board member of ImmunoMet and a founder of Rheos

569 Medicines. For the purpose of open access, the authors have applied a CC BY public

570 copyright license to any Author Accepted Manuscript version arising from this

571 submission. This work is licensed under a Creative Commons Attribution 4.0

572 International License. 


\section{FIGURE LEGENDS}

\section{Figure 1. mTORC1-deficient Zebrafish Are Hypersusceptible to Mm Infection.}

575 (A) Illustration of hindbrain ventricle (hbv) and caudal vein (cv) injection routes used in 576 this study.

577 Larvae were infected with $\sim 150 \mathrm{Mm}$ expressing tdTomato (B, C, E - J) or tdKatushka2

578 (D) fluorescent proteins via the caudal vein two days post fertilization (dpf).

579 (B) Overlaid micrographs of widefield mycobacterial fluorescence (Mm, red) and brightfield in $m t^{\text {fth } 178 / f h 178}$ or wildtype siblings $\left(m t o r^{+/+}\right)$four days post infection (dpi).

581 (C) Quantification of bacterial fluorescence (fluorescent pixel counts, FPC) in animals from $m t^{\text {fht }}{ }^{178 /+}$ incross 4 dpi. Symbols represent individual animals. Horizontal lines indicate mean values.

(D) Confocal micrographs of $m$ tor $^{\text {fh178/fht78 }}$ and a wildtype sibling expressing $\operatorname{Tg}$ (mpeg1:YFP) 4 dpi showing a granuloma in the wildtype animal and mycobacterial cording in the $m t^{\text {fh } 178 / f h 178}$ animal. Mm (magenta) and macrophages (green) are shown. Arrowheads indicate intracellular Mm. Bracket indicates Mycobacterial cording in animals from (E) $m t^{\text {fht } 178 /+}$ incross, (F) $m t^{\text {sa16755/+ }}$ incross, (G)

590 mtor $^{\text {fh178/+ }} \times$ mtor $^{\text {sa16755/+ }}$ complementation cross, and $(\mathrm{H})$ rptor $^{\text {sa11537/+ }}$ incross at $4 \mathrm{dpi}$,

591 and (I) rictora ${ }^{\text {sa15967// }}$; rictorb ${ }^{\text {sa18403/+ }}$ double heterozygote incross and (J) wildtype

592 animals treated with torin1 $(400 \mathrm{nM})$, rapamycin $(400 \mathrm{nM})$, or $0.5 \%$ DMSO (vehicle

593 control) 5 dpi. (E - J) Numbers within columns indicate animals per group. 
Scale bars, (B) $300 \mu \mathrm{m}$ and (D) $25 \mu \mathrm{m}$. Statistical analyses, (C) one-way ANOVA with Tukey's post-test and (E - J) Fisher's exact test. ${ }^{* \star *} p<0.0001$. Data shown are

596 representative of two or more independent experiments.

Figure 2. mTOR Deficiency Impairs Macrophage Development and Survival and

\section{Sensitizes Infected Macrophages to Mycobacterium-induced Cytotoxicity.} or tdKatushka2 $(\mathrm{F}-\mathrm{H})$ fluorescent proteins via the hindbrain ventricle $(\mathrm{A})$ or the caudal $602 \operatorname{vein}(B-N) 2$ dpf.

603 (A) Confocal micrographs showing serial imaging of granulomas in Tg(mfap4:tdTomato-CAAX) zebrafish treated with Rapamycin or DMSO. Mm (cyan) and macrophages (red) are shown.

(B) (Top) Illustration of macrophage counting region (shaded light blue). (Bottom) Numbers of macrophages in $\mathrm{Mm}$ - and mock-infected Raptor mutants and siblings expressing $T g$ (mpeg 1:tdTomato). Symbols indicate mean values for each group and error bars show Standard Error of the Mean (S.E.M.).

611 (D) Time-lapse confocal micrographs capturing the death of macrophage infected with

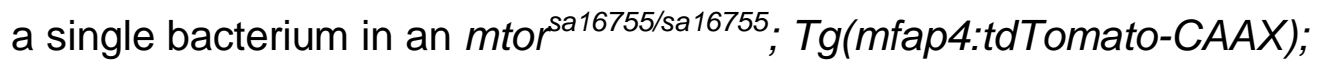

614 macrophage (magenta) are shown. Arrowheads indicate annexin V-decorated $615 \quad$ blebs. See Movie S1. 
616 (E - G) 6-hr time-lapse confocal microscopy of mtor $^{\text {fh178/fh178 }}$ and mTOR-sufficient

617 siblings expressing $T g(m p e g 1: Y F P) 2$ dpi. See Movie S2.

618 (E) Absolute numbers of infected macrophages in the imaged field.

619 (F) Percentage of dying macrophages in the imaged field.

620 (G) Relative mycobacterial burdens in dying macrophages of mtor $^{-/}$and mTOR-

621 sufficient fish. Bacterial volumes were normalized to values obtained from dying

622 cells in mTOR-sufficient controls for each imaging run.

$623(\mathrm{H})$ Widefield micrograph of parabiotic zebrafish comprised of conjoined wild-type

624 Tg(mpeg1:tdTomato) and mtor ${ }^{\text {fh178/fh178; } T g(m p e g 1: Y F P)}$ embryos 4 dpi.

625 (I) Macrophage counts (normalized to each genotype's initial counts) in the WT body

626 (top) and $m$ tor $^{-/-}$body (bottom) of WT - $m t^{-/-}$parabiont.

627 (J) Maximum intensity projections of infections in the WT body (top) and mtor $^{-/}$body

628 (bottom) of a WT - mtor ${ }^{-/-}$parabiont $4 \mathrm{dpi}$.

629 (K) Widefield micrograph of wild-type Tg(mpeg1:tdTomato) and mtor ${ }^{\text {fh178/+ }}$;

$630 \quad \operatorname{Tg}($ mpeg1:YFP) parabiont 4 dpi.

631 (L) Macrophage counts (normalized to each genotype's initial counts) in the WT body

632 (top) and mtor $^{+/-}$body (bottom) of WT - mtor $^{+/-}$parabiont.

633 (M) Maximum intensity projections of infections in the WT body (top) and mtor $^{+/-}$body 634 (bottom) of a WT - mtor $^{+/-}$parabiont 4 dpi.

635 Scale bars, (A) $25 \mu \mathrm{m},(\mathrm{D}) 10 \mu \mathrm{m},(\mathrm{H}, \mathrm{K}) 400 \mu \mathrm{m}$, and (J, M) $50 \mu \mathrm{m}$. Statistical analyses, $636(\mathrm{E}-\mathrm{G})$ two-tailed, unpaired Student's $t$ test. ${ }^{* *} p<0.01$. Time lapse data were pooled

637 from five (E and $F$ ) or three $(G)$ independent experiments. 


\section{Figure 3. mTOR Deficiency Impairs Mitochondrial Metabolism in Macrophages}

640 and Facilitates Mycobacterium-induced Mitochondrial Damage.

641 (A, B) $m t^{\text {fh } 178 / f h 178}$ and mTOR-sufficient siblings expressing $T g(m p e g 1: Y F P)$ were

642 infected intravenously with Mm expressing BFP2 on $2 \mathrm{dpf}$ and injected

643 intravenously with MitoTracker Red $\mathrm{CMH}_{2}$-Xros one day later.

644 (A) Confocal micrograph illustrating mROS detection in an infected animal.

645 Macrophages (green), Mm (blue), and mROS (magenta) are shown. Arrowheads

646 indicate mROS-producing infected cells. Scale bar, $20 \mu \mathrm{m}$.

647 (B) Quantification of MitoTracker Red $\mathrm{CMH}_{2}$-Xros mean fluorescence intensity (MFI) in

648 infected and uninfected macrophages of tor $^{-/}$animals and siblings at $1 \mathrm{dpi}$.

649 Symbols represent individual macrophages. Horizontal lines indicate mean values.

650 THP-1 macrophages were treated with torin1 or DMSO and infected with (C, D, G, H)

651 tdTomato- $(\mathrm{E}, \mathrm{F})$ or mWasabi-expressing $\mathrm{Mm}$ at a multiplicity of infection (MOI) of 1 (C -

$652 \mathrm{~F})$ or $3(\mathrm{G}, \mathrm{H})$.

653 (C) Flow cytometry plots of cell viability 2 dpi. Percentages of non-viable cells (FVD

654 eFluor $660^{+}$) in the infected and uninfected subpopulations are shown.

655 (D) Quantification of non-viable cells. Symbols represent values from individual wells,

$656 \quad$ and bars indicate mean values.

657 (E) Flow cytometry histograms of TMRE fluorescence 1dpi.

658 (F) TMRE geometric mean fluorescence intensities (GeoMFI) 1 dpi. Symbols

659 represent values from individual wells, and bars indicate mean values. 
660 (G) Flow cytometry histograms of cytochrome c (cyt c) fluorescence in infected viable cells (FVD eFluor 660') 7 hours post infection (7 hpi). Gate indicates population of cells that have released cyt c.

663

(H) Quantification of cyt c cow cells 7 hpi.

664 Statistical analyses, (B) one-way or (D, H) two-way ANOVA with Tukey's post-test. * $p<$ $6650.05,{ }^{* *} p<0.01,{ }^{* *} p<0.001$, and ${ }^{* * *} p<0.0001$. (A, B) Data are representative of two experiments. See also Figure S1.

Figure 4. Glycolysis Inhibition Impairs Mitochondrial Metabolism and Sensitizes Infected Macrophages to Mycobacterium-induced Cytotoxicity.

671 DMSO were infected with Mm expressing $(\mathrm{A}) \mathrm{BFP2}$ or $(\mathrm{B}, \mathrm{K})$ tdTomato $(\mathrm{MOI}=1)$.

672 (A) TMRE GeoMFI 1 dpi.

673 (B, C) Percentage of non-viable cells (FVD eFluor $660^{+}$) 1 dpi.

674 (D - H, J) Zebrafish were infected with 150 fluorescent Mm via the caudal vein.

675 (D) 5 dpi macrophage numbers in the body of mock- or Mm-infected Tg(mpeg1:YFP) zebrafish fish treated with $50 \mathrm{mM}$ 2DG or $0.5 \%$ DMSO.

677 (E, F) 6-hr time-lapse confocal microscopy of Tg(mpeg1:YFP) 3 dpi. (D) Absolute

678 numbers of infected macrophages in the imaged field. (E) Percentage of dying infected

679 macrophages in the imaged field. See Movie S3.

680 (G) Mycobacterial cording in 2DG- and DMSO-treated wildtype animals 5 dpi.

$681(\mathrm{H})$ Mycobacterial cording in wildtype animals treated with UK5099 (10 $\mu \mathrm{M})$ or $0.5 \%$ $682 \quad$ DMSO 5dpi. 
683 (I) Glucose-6 phosphate dehydrogenase (G6PD) activity in 5 dpf animals from $g 6 p d^{s a 24272 /+}$ incross.

(J) Mycobacterial cording in animals from $g 6 p d^{\text {sa24272/+ }}$ incross 5 dpi.

686 (K) ATP concentration per well containing $10^{6}$ THP-1 cells 1 dpi.

687 Symbols represent values from individual (A- C, K) wells or (D - J) animals. (A - C, I, K)

688 Bars and (D - F) horizontal lines indicate mean values. (G, H, J) Numbers within

689 columns indicate animals per group. Statistical analyses, one-way ANOVA with (A - C)

690 Sidak or (D, I) Tukey post-tests, (E, F) unpaired Student's $t$ test, or (G, H, J) Fisher's

691 exact test. ${ }^{*} p<0.05,{ }^{* *} p<0.01,{ }^{* * *} p<0.001$, and ${ }^{* * * *} p<0.0001$. (E, F) Time-lapse

692 data were pooled from two independent experiments. Data are representative of (A, G,

693 H) two independent experiments. See also Figure S2.

695 Figure 5. Deficiencies in mTOR, Glycolysis, and Mitochondrial Import of Pyruvate 696 Sensitize Macrophages to Mycobacterial ESAT-6-dependent Cytotoxicity.

697 (A) Cytochrome c release 7 hpi in THP-1 macrophages infected with BFP2-expressing $698 \quad \mathrm{WT}$ or $\triangle E S X-1 \mathrm{Mm}$ at $\mathrm{MOI}=3$.

699 (B) Percentage of dying cells (SYTOX Green ${ }^{+}$) during 4-hr time-lapse at 1 dpi with 700 tdTomato-expressing $\mathrm{WT}$ or $\triangle E S X-1 \mathrm{Mm}$ at $\mathrm{MOI}=1$. Values from uninfected $(\mathrm{U})$ $701 \quad$ and infected (I) cells from the same fields are shown. See Movie S4.

$702(\mathrm{C}-\mathrm{H})$ Zebrafish were infected with dose-matched inocula of tdTomato-expressing $\mathrm{Mm}$ 703 of the indicated strains via the caudal vein. 
704 (C) Percentage of dying infected macrophages in 6-hr time-lapse confocal microscopy of $m t^{\text {fht } 178 / h 178}$ and mtor-sufficient siblings expressing $\operatorname{Tg}(m p e g 1: Y F P) 2$ dpi. See Movie S5.

707

(D) Mycobacterial cording in $m$ tor $^{\text {fh178/fht78 }}$ animals and mtor-sufficient siblings $4 \mathrm{dpi}$. 5 dpi.

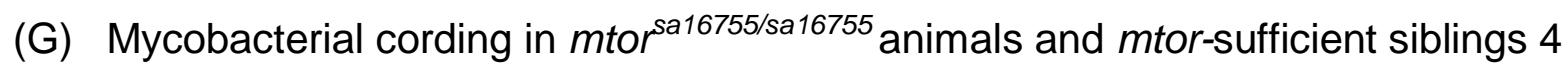
dpi. See also Figure S3.

(H) Mycobacterial cording in mtor $^{\text {fh178/fh } 178}$ animals and mtor-sufficient siblings 4 dpi with $\triangle$ esxA Mm complemented with wild-type or point mutant Mtb esxA.

714 Symbols represent values from individual (A) wells, (B) imaging fields, or (C) animals.

715 (A, B) Bars and (C) horizontal lines indicate mean values. (D - H) Numbers within

716 columns indicate animals per group. Statistical analyses, (A - C) one-way ANOVA with

717 Sidak's post-test or (D - H) Fisher's exact test. ${ }^{*} p<0.05,{ }^{* *} p<0.01,{ }^{\star * *} p<0.001$, and

$71{ }^{* * * *} p<0.0001$. (B, D) Data are representative of two experiments. Zebrafish time-lapse 719 data were pooled from three experiments.

Figure 6. ESX-1 Mediates Mitochondrial Damage in mTOR-deficient Macrophages

722 Downstream of Its Involvement in Phagosomal Permeabilization.

723 (A - E) Torin1- and DMSO-treated THP-1 macrophages were infected with tdTomato-

724 expressing WT or $\triangle E S X-1 \mathrm{Mm}$ at $\mathrm{MOI}=3$ and treated with prazosin $(\mathrm{PRZ}, 20 \mu \mathrm{M})$ for 7

725 hrs. See also Figure S4.

726 (A) Confocal micrographs of Galectin-8 (GAL8) immunofluorescence (green) and Mm 727 fluorescence (magenta) in THP-1 macrophages 7 hpi. GAL8 foci associated with 
Mm (filled arrowheads) or not associated with Mm (open arrowheads) are shown.

729 Scale bar, $20 \mu \mathrm{m}$.

730 (B) Percentage of macrophages with GAL8-associated Mm foci.

731 (C) Percentage of Mm volume associated with GAL8 foci $7 \mathrm{hpi}$.

732 (D) Percentage of cells that have released cytochrome c 7 hpi.

733 (E) $m$ tor $r^{\text {sa16755/sa16755 }}$ fish and mtor-sufficient siblings were infected with 90 fluorescent

$734 \quad$ Mm via the hindbrain ventricle on 2 dpf. On 1 and 2 dpi, fish were injected with $\sim 3$

$735 \mathrm{~nL}$ of $300 \mu \mathrm{M} \mathrm{PRZ}$ or $1 \%$ DMSO into the hindbrain ventricle. Graph indicates the

736 percentage of animals with mycobacterial cording 3 dpi.

737 Symbols represent values from individual $(B, C)$ imaging fields or (D) individual wells.

$738(B, C)$ Horizontal lines and (D) bars indicate mean values. (E) Numbers within columns

739 indicate animals per group. Statistical analyses, (B, D) one-way ANOVA with Sidak's

740 post-test or (E) Fisher's exact test. ${ }^{*} p<0.05,{ }^{* *} p<0.01,{ }^{* *} p<0.001$, and ${ }^{* \star * *} p<$

7410.0001.

742

743 Figure 7. Cartoon Model Depicting the Role of mTOR-associated Mitochondrial

744 Energy Metabolism in Protection from Mycobacterial ESAT-6-induced

745 Mitochondrial Damage and Cytotoxicity. 
MATERIALS AND METHODS

RESOURCE AVAILABILITY

750 Additional information and requests for resources and reagents should be directed to

751 and will be fulfilled by the Lead Contact, Lalita Ramakrishnan (lalitar@mrc-

752 Imb.cam.ac.uk).

754 EXPERIMENTAL MODEL AND SUBJECT DETAILS

\section{Ethics Statement}

756 Zebrafish husbandry and experiments were carried out in compliance with guidelines

757 from the UK Home Office and the US Public Health Service Policy on Human Care and

758 Use of Laboratory Larvae using protocols approved by the Animal Welfare and Ethical

759 Review Body of the University of Cambridge and the Institutional Animal Care and Use

760 Committee of the University of Washington, respectively.

\section{Zebrafish Husbandry and Infections}

763 Zebrafish of the wild-type AB strain (Zebrafish International Resource Center, ZIRC), TL

764 strain (ZIRC), or of mixed $A B / T L$ backgrounds were used in experiments (Table S2).

765 The $\operatorname{Tg}\left(\right.$ mpeg1:YFP) ${ }^{w 200}, \operatorname{Tg}$ (mpeg1:Brainbow) $^{\text {w201 }}$ (described as mpeg1:tdTomato),

766 Tg(mfap4:tdTomato-CAAX) ${ }^{x t 6}, \operatorname{Tg}(C M V \text { :EGFP-map1/c3b })^{z f 155}$, and Tg(ubb:secA5-

$767 Y_{Y F P}{ }^{\text {cu34 }}$ (this work) fluorescent reporter lines were maintained in the $\mathrm{AB}$ strain (He et

768 al., 2009; Pagan et al., 2015; Roca and Ramakrishnan, 2013; Walton et al., 2015)

769 (Table S2). The tor $^{\text {fh } 178}$ mutant line (this work) was generated and maintained in the 


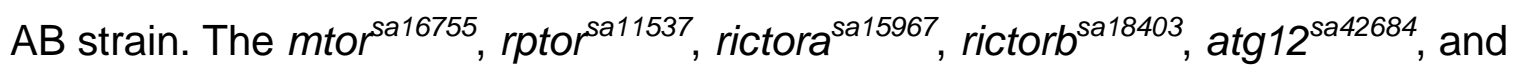

$771 g 6 p d^{\text {sa24272 }}$ mutant lines (Wellcome Trust Sanger Institute) (Kettleborough et al., 2013)

772 were generated in the TL strain and maintained in either the TL strain (rictora ${ }^{\mathrm{sa15967}}$,

773 rictorb $^{\text {sa18403 }}$, and $g 6 p d^{\text {sa24272 }}$ ) or mixed AB/TL backgrounds (mtor ${ }^{\text {sa16755 }}$, rptor ${ }^{\text {sa11537, }}$, and

$774 \operatorname{atg} 12^{\text {sa42684}}$ ) (Table S2). Zebrafish of the WIK strain (ZIRC) were used to map mtor ${ }^{\text {fh178. }}$

775 Zebrafish were maintained in buffered reverse osmotic water systems under a 14-hr

776 light/10-hr dark cycle. Zebrafish larvae were fed paramecia twice daily, while juvenile

777 and adult zebrafish were fed at least twice a day with dry food and brine shrimp.

778 Zebrafish embryos were collected and cultured in reverse osmosis water containing

$7790.18 \mathrm{~g} / \mathrm{L}$ Instant Ocean Salt supplemented with $0.25 \mu \mathrm{g} / \mathrm{mL}$ methylene blue at $28.5^{\circ} \mathrm{C}$. On

$7801 \mathrm{dpf}$, embryos to be used in experiments were transferred to $0.5 \mathrm{x}$ E2 medium $(7.5 \mathrm{mM}$

$781 \mathrm{NaCl}, 0.25 \mathrm{mM} \mathrm{KCl}, 0.5 \mathrm{mM} \mathrm{MgSO}_{4}, 0.075 \mathrm{mM} \mathrm{KH}_{2} \mathrm{PO}_{4}, 0.025 \mathrm{mM} \mathrm{Na}_{2} \mathrm{HPO}_{4}, 0.5 \mathrm{mM}$

$782 \mathrm{CaCl}_{2}$, and $0.35 \mathrm{mM} \mathrm{NaHCO}_{3}$ ) supplemented with $0.003 \%$ PTU (1-phenyl-2-thiourea,

783 Sigma) to inhibit melanin synthesis.

$784 \quad$ For infections, 2 dpf larvae of undetermined sex (due to early developmental

785 stage) were dechorionated manually or with $\leq 0.5 \mathrm{mg} / \mathrm{mL}$ pronase (Sigma-Aldrich) and

786 then anesthetized with fish water containing $0.025 \%$ tricaine (Sigma-Aldrich). Larvae

787 were injected via the caudal vein or the hindbrain ventricle using single-cell suspensions

788 of Mm of known titer to deliver $\sim 100-250$ bacteria per $3-5 \mathrm{~nL}$ injection as previously

789 described (Takaki et al., 2013). Phenol red sodium salt $(\leq 1 \% \mathrm{w} / \mathrm{v}$ diluted in PBS,

790 Sigma-Aldrich) was used as an injection tracer. Inoculums were confirmed by injecting

791 onto Middlebrook 7H10 agar plates (supplemented with oleic acid, albumin, dextrose,

792 and Tween-80 plus hygromycin B or kanamycin, as appropriate. For experiments with 
793 mutant lines, wild-type and heterozygous siblings were used as comparators, animals

794 were genotyped after data acquisition. In experiments involving drug treatments,

795 infected animals were randomly assigned to treatment groups. Unless indicated,

796 treatments (Table S2) were initiated immediately after infection via soaking, and drug

797 exposure was maintained until the experimental endpoint. UK5099 (Cambridge

798 Bioscience) was changed daily.

\section{THP-1 Macrophage Culture and Infections}

801 Monocytic human THP-1 cells (ATCC) were grown at $37^{\circ} \mathrm{C}, 5 \% \mathrm{CO}_{2}$ in $\mathrm{RPMI}$ medium

802 (Sigma-Aldrich) supplemented with 10\% FBS (Gibco), L-glutamine (Gibco), Penicillin, 803 and Streptomycin (complete RPMI) (Table S2). To differentiate THP-1 cells into

804 macrophages, cells were seeded at $5 \times 10^{5}$ cells $/ \mathrm{mL}$ in flat-bottomed tissue culture

805 plates $\left(2.5 \times 10^{5}\right.$ cells/well of 24 -well plate $)$ and stimulated with $100 \mathrm{nM}$ PMA Phorbol 12 -

806 myristate 13-acetate (Sigma-Aldrich) for two days. The resulting adherent cells were

807 then washed with complete RPMI and rested for two days prior to infection. For

808 microscopy experiments, cells were plated on optical bottom plates (Perkin Elmer or

809 MatTek). Cells were pre-incubated overnight with pharmacological compounds or

810 matching concentrations of vehicle ( $\leq 0.5 \%$ DMSO). On the infection day, cells were

811 washed with antibiotic-free complete RPMI and infected with single-cell suspensions of

$812 \mathrm{Mm}$ or Mtb for five hours in a $33^{\circ} \mathrm{C}, 5 \% \mathrm{CO}_{2}$ incubator (for $\mathrm{Mm}$ ) and $37^{\circ} \mathrm{C}, 5 \% \mathrm{CO}_{2}$

813 incubator (for Mtb). After infection, cells were washed with antibiotic-free complete

814 RPMI supplemented with the corresponding pharmacological compounds and returned

815 to the appropriate incubators. For experiments with the leucine and pantothenate 
816 double auxotroph $\triangle l e u D \Delta p a n C D$ Mtb, complete RMPI was also supplemented with 0.05

$817 \mathrm{mg} / \mathrm{mL}$ L-leucine and $0.024 \mathrm{mg} / \mathrm{mL}$ calcium pantothenate (Sigma-Aldrich). Multiplicity of

818 infection was determined by calculating the number of mycobacterial colony forming

819 units (CFU) per cells plated in each well.

\section{METHOD DETAILS}

\section{Bacterial Strains}

M. marinum $\mathrm{M}$ strain (ATCC \#BAA-535) and its mutant derivatives $\triangle E S X-1$,

$824 \Delta m m p L 7, \Delta e r p, \operatorname{marP}:$ :tn, and $\Delta$ esxA expressing BFP2, mWasabi, tdTomato, or

825 tdKatushka2 under the control of the msp12 promoter (Cambier et al., 2014b; Cosma et

826 al., 2006; Levitte et al., 2016; Osman et al., 2022; Takaki et al., 2013) were grown at

$82733^{\circ} \mathrm{C}$ under hygromycin B (Cambridge Bioscience) or kanamycin (Sigma-Aldrich)

828 selection in Middlebrook 7H9 medium (BD Difco) supplemented with oleic acid, albumin,

829 dextrose, and Tween-80 (Sigma-Aldrich) (Takaki et al., 2013) (Table S2). M.

830 tuberculosis $\triangle l e u D \Delta p a n C D \mathrm{mc}^{2} 6206$ expressing msp12:tdTomato was grown at $37^{\circ} \mathrm{C}$

831 under hygromycin B and kanamycin selection in Middlebrook 7H9 medium

832 supplemented with oleic acid, albumin, dextrose, Tween-80, catalase, and $0.05 \mathrm{mg} / \mathrm{mL}$

833 L-leucine and $0.024 \mathrm{mg} / \mathrm{mL}$ calcium pantothenate (Sigma-Aldrich) (Roca et al., 2019;

834 Sampson et al., 2011).

835

\section{Zebrafish Genotyping}

837 DNA from adult fin clips or whole larvae was extracted using the HotSHOT method

838 (Truett et al., 2000). Animals were genotyped by High Resolution Melt Analysis (HRMA) 
839 of PCR products (Garritano et al., 2009) or by Kompetitive Allele-Specific PCR (KASP)

840 assay (LGC Biosearch) (He et al., 2014) in a CFX Connect thermocycler (BioRad)

841 (Table S2). DNA from 2DG-treated animals was purified with AMPure XP beads

842 (Beckman Coulter) according to the manufacturer's instructions prior to PCR.

844 Mapping of mtor $^{\text {fh178 }}$

845 Zebrafish carrying the fh178 allele were outcrossed to the WIK strain for mapping as

846 previously described (Tobin et al., 2010). fh178 was mapped to chromosome 8 initially

847 between the markers z7370 and z14670. Further mapping defined a critical two-gene

848 interval that included mtor and qars 1 to the right of a single nucleotide polymorphism in

849 the 3' end of angpt/7 (1 recombination in 302 meioses) and to the left of a polymorphism

850 in intron 2 of ogg1 (2 recombinations in 300 meioses). Sequencing of cDNAs isolated

851 from mutants and wild-type animals in the critical region identified a stop codon in exon

85224 of mtor in the mutant but not wild-type animals. This mutation segregated absolutely

853 with the fh178 mutants (no recombinants).

855 Creation of $\mathrm{Tg}(u b b: \operatorname{secA5}-\text { YFP) })^{\text {cu34 }}$

856 The Tol2 ubb:secA5-YFP plasmid was assembled from PCR-amplified fragments of

857 pDestTol2pA2_ubi:EGFP (RRID: Addgene 27323) (Mosimann et al., 2011) and pBH-

858 UAS-secA5-YFP (RRID: Addgene 32359) (van Ham et al., 2010) the Gibson Cloning kit

859 (New England Biolabs) (Table S2). Correct plasmid assembly was confirmed by

860 diagnostic PCR of joined segments and by Sanger sequencing. Tol2 transposase

861 (RRID: Addgene 51818) (Khattak et al., 2014) was in vitro transcribed with the T7 
862 mMessage/mMachine kit (ThermoFisher) according to the manufacturer's instructions

863 (Table S2). Tol2 ubb:secA5-YFP plasmid and transposase mRNA were co-injected into

864 one-cell embryos of the wild-type AB strain as previously described (Suster et al.,

865 2011). $G_{0}$ larvae expressing the transgene were identified by fluorescence microscopy

866 and raised to adulthood. Potential founders were identified through pairwise crosses of

$867 \mathrm{G}_{0}$ adults and non-transgenic $A B$ fish. $A$ single $F_{1}$ transgenic animal was used to

868 establish the line.

\section{Parabiosis}

871 Parabiosis of zebrafish embryos was performed as previously described (Demy et al.,

872 2013). Briefly, blastulae derived from synchronized $\left.m t^{f h 178 /+} ; \operatorname{Tg}^{\text {(mpeg1:YFP }}\right)^{\text {w200 }}$ and

$873 \mathrm{Tg}$ (mpeg1:tdTomato) incrosses were manually dechorionated, paired, scratched with an

874 aluminum silicate microinjection needle, and allowed to fuse in high-calcium Ringer's

875 solution (116 mM NaCl, $2.9 \mathrm{mM} \mathrm{KCl}, 10 \mathrm{mM} \mathrm{CaCl}_{2}, 5 \mathrm{mM} \mathrm{HEPES}$, pH 7.2)

876 supplemented with $50 \mathrm{U} / \mathrm{mL}$ penicillin and $50 \mathrm{U} / \mathrm{mL}$ streptomycin. Only parabiotic pairs

877 fused at the head, devoid of gross morphological abnormalities, and sharing blood

878 circulation were used in experiments. On $2 \mathrm{dpf}$, each parabiotic animal was infected via

879 the caudal vein to ensure similar distribution of mycobacteria between the pair's caudal

880 hematopoietic tissues. The bodies of each parabiotic pair were micro-dissected and

881 individually genotyped after completion of the experiment.

882

883 Microscopy and Image Analyses 
884 Widefield fluorescence microscopy was performed as previously described (Takaki et

885 al., 2013). Bacterial burdens were determined with a Nikon Eclipse Ti-E inverted

886 microscope using a 4x objective and running a fluorescent pixel counts macro in ImageJ

887 (National Institutes of Health) (Takaki et al., 2013). Macrophages were counted

888 manually with a Nikon Eclipse E600 upright microscope using a 20x objective. For serial

889 imaging of individual zebrafish, larvae were housed individually in 96-well plates under

890 standard husbandry conditions.

891 Laser scanning confocal microscopy was performed as described (Pagan et al.,

892 2015). Larvae were anesthetized in PTU-supplemented 0.5x E2 medium containing

$893 \quad 0.025 \%$ tricaine and embedded in $2 \%$ low melt agarose (TopVision) on optical-bottom

894 plates (MatTek). Imaging was performed with a Nikon A1R confocal microscope using

$89520 x$ or $40 x$ objectives and the galvano or resonant scanners. $0.9-2 \mu \mathrm{m}$ optical sections

896 were combined to generate 15 - $60 \mu \mathrm{m}$ z stacks. For time-lapse microscopy of zebrafish

897 larvae, imaging was done at $27^{\circ} \mathrm{C}$ using a microscope incubator (Okolab), using

898 acquisition intervals of 2 - 5 minutes for 6 hours. For serial imaging of individual

899 zebrafish, larvae were removed from the agarose using jeweler's forceps and housed

900 individually under standard husbandry conditions. For time-lapse microscopy of Mm-

901 infected THP-1 cells, samples were imaged at $33^{\circ} \mathrm{C}, 5 \% \mathrm{CO}_{2}$ in antibiotic-free, phenol-

902 free complete RPMI containing 50 nM SYTOX Green nucleic acid stain (Invitrogen) for 4

903 hours.

904 The acquired images were processed using the denoising feature in the

905 Elements software (Nikon). The surface rendering feature of Imaris (Bitplane) was used

906 to quantify intramacrophage bacterial burdens and mROS levels; the number of acridine 
907 orange foci in the midbrain and LC3 puncta in neuromasts, clusters of mechanosensory

908 hair cells of the lateral line in fish; and the frequencies of galectin-8-associated Mm per

909 imaging field (Thomas et al., 2015). The numbers of dying macrophages and

910 macrophages with galectin-8-decorated Mm foci were counted manually.

911

\section{Acridine Orange Staining}

913 Zebrafish larvae were transferred to $1.5 \mathrm{~mL}$ microcentrifuge tubes and stained with 1

$914 \mathrm{~mL}$ of $2.4 \mu \mathrm{g} / \mathrm{mL}$ acridine orange in $0.5 x \mathrm{E} 2$ medium with PTU for 30 minutes with

915 rotation and protected from light (Roca et al., 2019). Stained larvae were then rinsed in

916 0.5x E2 medium with PTU and imaged by confocal microscopy.

917

\section{MitoTracker Red $\mathrm{CMXH}_{2}-\mathrm{Xros}$ Staining}

919 Zebrafish larvae were injected with $\sim 1 \mathrm{~nL}$ of $10 \mathrm{mM}$ MitoTracker Red $\mathrm{CMXH}_{2}-\mathrm{Xros}_{\text {via }}$

920 the caudal vein immediately prior to imaging by confocal microscopy (Roca and

921 Ramakrishnan, 2013).

922

923 Galectin-8 Immunostaining

924 The staining procedure was performed as previously described (Osman et al., 2020).

925 THP-1 cells were fixed in PFA solution (4\% w/v paraformaldehyde in PBS) at room

926 temperature (RT) for at least 30 minutes. Cells were then washed twice with PBS and

927 incubated in permeabilization/block (PB) solution $(0.1 \%$ Triton-X $100,1 \%$ bovine serum

928 albumin in PBS) for 30 minutes at RT, and subsequently stained with goat anti-human

929 galectin-8 antibody (R\&D Systems) diluted in PB solution overnight at $4^{\circ} \mathrm{C}$. The 
930 following day, cells were washed three times with PBS and stained with AlexaFluor488-

931 conjugated donkey anti-goat IgG (ThermoFisher) in PB solution for one hour at RT.

932 Cells were then washed three times with PBS and maintained in PBS for imaging by

933 confocal microscopy.

934

935 Flow Cytometry

936 THP-1 macrophages were stained with Fixable Viability Dye (FVD) eFluor 660

937 (eBioscience), detached with Accutase (Sigma-Aldrich), and transferred to polystyrene

938 FACS tubes (Sarstedt). Single-cell suspensions were washed with MACS buffer $(0.5 \%$

939 bovine serum albumin, $2 \mathrm{mM}$ EDTA in PBS, ph7.2) at $290 \mathrm{xg}$ for 5 minutes at $4^{\circ} \mathrm{C}$, and

940 in most cases, fixed in $4 \%$ PFA solution overnight at $4^{\circ} \mathrm{C}$. The following day, samples

941 were washed with and resuspended in MACS buffer. Data were acquired in LSRII or

942 Fortessa flow cytometers (BD Biosciences) and analyzed in FlowJo 10 (TreeStar),

943 gating on single-cell events.

944

945 TMRE Staining

946 THP-1 macrophages were incubated with 100 nM tetramethylrhodamine, ethyl ester

947 (TMRE) (Abcam) for 20 minutes in a $37^{\circ} \mathrm{C}, 5 \% \mathrm{CO}_{2}$ incubator according to the

948 manufacturer's instructions. Cells were then washed with complete RPMI media,

949 stained with FVD eF660, and processed for flow cytometry as above but without

950 fixation.

951

952 Cytochrome c Release Assay 
953 The cytochrome c release assay was adapted from Lienard, et al. (Lienard et al., 2020).

9547 hours after infection with tdTomato-expressing Mm, cells were stained with Fixable

955 Viability Dye (FVD) eFluor 660 (eBioscience), detached with Accutase (Sigma-Aldrich),

956 transferred to polystyrene FACS tubes (Sarstedt), and washed first with ice-cold MACS

957 buffer (0.5\% bovine serum albumin, 2mM EDTA in PBS, ph7.2) and then ice-cold PBS

958 by centrifugation at $290 \times \mathrm{g}$ for 5 minutes at $4^{\circ} \mathrm{C}$. Cells were incubated in ice-cold

959 permeabilization buffer $(50 \mu \mathrm{g} / \mathrm{mL}$ digitonin, $100 \mathrm{mM} \mathrm{KCl})$ for 5 minutes and immediately

960 fixed in 4\% PFA solution to stop the permeabilization reaction. Cells were then

961 centrifuged as above and fixed overnight in $4 \%$ PFA solution at $4^{\circ} \mathrm{C}$. Samples were

962 analyzed by flow cytometry by gating on the viable, infected (FVD eFluor 660

963 tdTomato ${ }^{+}$) single-cell events.

964

\section{ATP Quantification}

966 ATP levels were quantified by fluorimetry in a CLARIOStar Plus (BMG Labtech)

967 microplate reader using the ATP Assay Kit (Abcam) according to the manufacturer's

968 instructions. $10^{6}$ THP-1 macrophages were analyzed per replicate.

\section{Measurement of G6PD Activity}

971 Individual $5 \mathrm{dpf}$ larvae from an $g 6 p d^{\text {sa24272/+ }}$ incross were lysed using Mammalian Cell

972 Lysis Buffer (Abcam). A small amount of each lysate was used to genotype the larvae.

973 G6PD activity in individual larvae was determined by fluorimetry in a CLARIOStar Plus

974 (BMG Labtech) microplate reader using the G6PD Activity Assay Kit (Abcam) according

975 to the manufacturer's instructions. 


\section{Metabolic Analyses}

978 For metabolite quantification, THP-1 cells $\left(2 \times 10^{6}\right.$ cells/sample, four

979 replicates/condition) were washed in ice-cold PBS, and metabolites were extracted with

$980150 \mu \mathrm{L}$ extraction buffer (50:30:20, methanol:acetonitrile:water) cooled on dry ice for 30

981 minutes beforehand. Samples were then centrifuged at maximum speed for 10 minutes

982 at ${ }^{\circ} \mathrm{C}$ to remove protein debris, and supernatants were stored at $-80^{\circ} \mathrm{C}$ until acquisition.

983 Liquid chromatography-mass spectrometry (LC-MS) was carried out using an Agilent

9841290 Infinity II UHPLC in line with a Bruker Impact II QTOF operating in negative ion

985 mode, as previously described (Edwards-Hicks et al., 2020). LC separation was

986 performed on a Phenomenex Luna propylamine column (50 x 2 mm, $3 \mu \mathrm{m}$ particles)

987 using a solvent gradient of $100 \%$ buffer B (5 mM ammonium carbonate in $90 \%$

988 acetonitrile) to $90 \%$ buffer $\mathrm{A}\left(10 \mathrm{mM} \mathrm{NH}_{4}\right.$ in water). Flow rate was from 1000 to 750

$989 \mu \mathrm{L} / \min$. Autosampler temperature was $5^{\circ} \mathrm{C}$, and injection volume was $2 \mu \mathrm{L}$. Metabolites

990 were quantified using AssayR (Wills et al., 2017). The hierarchical clustering heatmap

991 was generated in MetaboAnalyst 5.0 using Euclidean distance measure, the Ward

992 clustering algorithm, and autoscale feature standarization (Pang et al., 2021).

993 Metabolic flux measurements were carried out with the Seahorse XFp Glycolytic

994 Rate Assay and Cell Mito Stress Test kits (Agilent) according to the manufacturer's

995 instructions. On the day of the assay, $4 \times 10^{4}$ differentiated THP-1 macrophages were

996 seeded per well of the XFp cartridge, allowed to adhere for 2 hours in a $37^{\circ} \mathrm{C}, 5 \% \mathrm{CO}_{2}$

997 incubator, and then incubated with assay medium (XF DMEM medium with $10 \mathrm{mM}$

998 glucose and $2 \mathrm{mM}$ glutamine but without sodium pyruvate (Agilent)) for 45 - 60 minutes 
999 in a non- $\mathrm{CO}_{2} 37^{\circ} \mathrm{C}$ incubator prior to data acquisition in a Seahorse XFp analyzer

1000 (Agilent).

1001

1002 Statistical Analyses

1003 Statistical analyses were performed on Prism (GraphPad) with each statistical test and

1004 number of samples per group indicated in the corresponding figure legend. Not

1005 significant, $p>0.05 ;{ }^{*} p<0.05 ;{ }^{* *} p<0.01 ;{ }^{* * *} p<0.001 ;{ }^{* *} p<0.0001$.

1006

1007

1008

1009 


\section{SUPPLEMENTARY FIGURES, TABLES, AND MOVIES}

1011 Figure S1. Inhibition of Autophagic Cell Death, Mitochondrial Apoptosis, or TNF-

1012 associated Necrosis Does Not Prevent Mycobacterium-induced Macrophage

1013 Death in mTOR-deficient Animals, Related to Figures 2 and 3.

1014 (A) Confocal micrographs of LC3 clustering in neuromasts, clusters of

1015 mechanosensory cells of the fish lateral line, from atg $12^{\text {sa42684 }}$ incross fish

1016 expressing $\operatorname{Tg}(C M V: I c 3 b-G F P) 5$ dpf. GFP fluorescence (top) and surface-

1017 rendered puncta (bottom) are shown. Scale bar, $10 \mu \mathrm{m}$.

1018 (B) Number of LC3 puncta per neuromast.

1019 (C) Mycobacterial cording in rapamycin- or DMSO-treated atg12 $2^{\text {sa42684 }}$ incross fish 5

1020 dpi.

1021 (D) Confocal micrographs of acridine orange (AO) staining (green) and surfacerendered puncta (magenta) in the midbrain of casp9sa incross fish $3 \mathrm{dpf}$. Scale

1023 bar, $50 \mu \mathrm{m}$.

1024 (E) Number of AO puncta in the midbrain.

1025 (F) Mycobacterial cording in rapamycin- or DMSO-treated casp9 $9^{\text {sa11164 }}$ incross fish 5 1026 dpi.

1027 (G) Illustration of necrosis pathway induced by mycobacterial infection plus excess 1028 TNF and pharmacological interventions tested.

$1029(\mathrm{H}, \mathrm{I})$ Mycobacterial cording in $m t^{\mathrm{fh}} \mathrm{r}^{178 / \mathrm{H}}$ incross fish treated with $(\mathrm{H})$ necrostatin-1, (I) $1030 \quad$ nifedipine $(5 \mu \mathrm{M})$, diltiazem $(10 \mu \mathrm{M})$, or $0.5 \%$ DMSO 4 dpi.

1031 Symbols represent individual (B) neuromasts or (E) animals. (B, E) Horizontal lines

1032 indicate mean values. (C, F, H, I) Numbers within columns indicate animals per group. 
$1033(\mathrm{~B}, \mathrm{E})$ one-way ANOVA with Tukey's post-test. ${ }^{*} p<0.05,{ }^{* \star *} p<0.001$, and ${ }^{* \star * *} p<$ 10340.0001.

Figure S2. mTOR Inhibition Impairs Glycolysis and Mitochondrial Metabolism,

\section{Related to Figure 4.}

1038 (A) Metabolite profiles of THP-1 macrophages 1.5 days after treatment with torin1

$1039(400 \mathrm{nM}), 2 \mathrm{DG}(5 \mathrm{mM})$, or $0.5 \%$ DMSO. Heat map scale indicates relative $\log _{2}$

1040 expression levels. See also Table S1.

1041 (B) Diagram of Glycolytic Proton Efflux Rate (PER) assay.

1042 (C) PER kinetics of THP-1 macrophages treated with torin1 or DMSO for 1.5 days.

1043 (D) Basal and compensatory glycolytic PER.

1044 (E) Diagram of Mitochondrial Oxygen Consumption Rate (OCR) assay.

1045 (F) OCR kinetics of THP-1 macrophages treated with torin1 or DMSO for 1.5 days.

1046 (G) Modular analysis of mitochondrial OCR.

$1047(\mathrm{H})$ OCR kinetics of THP-1 macrophages treated with 2DG or DMSO.

1048 (I) Modular analysis of mitochondrial OCR.

1049 (J) Relative ATP levels in THP-1 macrophage cultures 1.5 days after treatment.

1050 Symbols represent $(B, C, E, F, H)$ mean values or (J) individual wells. (D, G, I, J) Bars

1051 indicate mean values. (D, G, I) Error bars depict Standard Deviation. (B, E) Arrows

1052 indicate the time of compound injection. Abbreviations: rotenone plus antimycin A (Rot + 1053 AA), 2DG, oligomycin (Oligo), carbonyl cyanide 4-(trifluoromethoxy)phenylhydrazone

1054 (FCCP), compensatory glycolysis (Comp), spare respiratory capacity (SRC). 
Figure S3. Phagosome-damaging Capacity of ESX-1-competent Mycobacteria,

\section{Related to Figure 5.}

1058 (A) Confocal micrographs of Galectin-8 (GAL8) immunofluorescence (green) and Mm 1059 fluorescence (magenta) in THP-1 macrophages infected with the indicated Mm

(B) Percentage of macrophages with foci of GAL8-associated Mm. Symbols represent values from individual imaging fields. Horizontal bars indicate mean values. One-

Figure S4. Prazosin Damages Lysosomes of THP-1 Macrophages, Related to

\section{Figure 6.}

1069 Confocal micrographs of GAL8 immunofluorescence 7 hours after treatment with 1070 prazosin (PRZ, $20 \mu \mathrm{M})$ or 0.5\% DMSO. Arrowheads indicate GAL8 puncta. Scale bar, $107120 \mu \mathrm{m}$.

1074 DMSO, Related to Figure S2.

1076 Movie S1. Annexin V Labeling of a Dying, Mm-infected Macrophage in an mTOR

1077 Mutant Animal, Related to Figure 2. Time-lapse confocal microscopy of a dying,

1078 infected macrophage in an mtor ${ }^{\text {sa16755/sa16755; }}$ Tg(mfap4:tdTomato-CAAX); 
1079 Tg(ubib:secA5-YFP) animal 2 dpi. Mm (blue), secreted annexin V-YFP (green), and

1080 macrophage (magenta) are shown.

1081

1082 Movie S2. Death of Mm-infected Macrophages in mTOR Mutant Animals, Related

1083 to Figure 2. Time-lapse confocal microscopy of $m t^{\text {fht }}{ }^{\text {178/fh178 }}$ animals and mTOR-

1084 sufficient siblings expressing Tg(mpeg1:YFP), 2 dpi. Mm (magenta) and macrophages

1085 (green) are shown. Arrows indicate dying infected macrophages.

1086

1087 Movie S3. Death of Mm-infected Macrophages in 2DG-treated Animals, Related to

1088 Figure 4. Time-lapse confocal microscopy of $\operatorname{Tg}(m p e g 1: Y F P)$ animals treated with 2DG

1089 or DMSO, 3 dpi. Mm (magenta) and macrophages (green) are shown. Arrows indicate

1090 dying infected macrophages.

1091

1092 Movie S4. Torin1-treated THP-1 Macrophages Tolerate $\Delta E S X-1$ Infection, Related

1093 to Figure 5. Time-lapse confocal microscopy of THP-1 macrophage treated with torin1

1094 or DMSO and infected with WT or $\triangle E S X-1 \mathrm{Mm}, 1$ dpi. Mm (magenta) and SYTOX

1095 Green-labeled dying cells (green). Arrows indicate dying infected macrophages.

1097 Movie S5. Macrophages of mTOR Mutant Animals Tolerate $\triangle E S X-1$ Infection,

1098 Related to Figure 5. Time-lapse confocal microscopy of $m$ tor $^{\text {fh178/fh } 178}$ animals and

1099 mTOR-sufficient siblings expressing $\mathrm{Tg}($ mpeg1:YFP) infected with WT or $\triangle E S X-1 \mathrm{Mm}$,

11002 dpi. Mm (magenta) and macrophages (green) are shown. Arrows indicate dying

1101 infected macrophages. 


\section{REFERENCES}

1103 Amaral, E.P., Costa, D.L., Namasivayam, S., Riteau, N., Kamenyeva, O., Mittereder, L.,

1104 Mayer-Barber, K.D., Andrade, B.B., and Sher, A. (2019). A major role for ferroptosis in

1105 Mycobacterium tuberculosis-induced cell death and tissue necrosis. J Exp Med 216,

1106 556-570. 10.1084/jem.20181776.

1107 Armstrong, J.A., and Hart, P.D. (1971). Response of cultured macrophages to

1108 Mycobacterium tuberculosis, with observations on fusion of lysosomes with

1109 phagosomes. J Exp Med 134, 713-740. 10.1084/jem.134.3.713.

1110 Augenstreich, J., Arbues, A., Simeone, R., Haanappel, E., Wegener, A., Sayes, F., Le

1111 Chevalier, F., Chalut, C., Malaga, W., Guilhot, C., et al. (2017). ESX-1 and phthiocerol

1112 dimycocerosates of Mycobacterium tuberculosis act in concert to cause phagosomal

1113 rupture and host cell apoptosis. Cell Microbiol 19. 10.1111/cmi.12726.

1114 Augenstreich, J., Haanappel, E., Ferre, G., Czaplicki, G., Jolibois, F., Destainville, N.,

1115 Guilhot, C., Milon, A., Astarie-Dequeker, C., and Chavent, M. (2019). The conical shape

1116 of DIM lipids promotes Mycobacterium tuberculosis infection of macrophages. Proc Natl

1117 Acad Sci U S A 116, 25649-25658. 10.1073/pnas.1910368116.

1118 Bao, Y., Wang, L., and Sun, J. (2021). A Small Protein but with Diverse Roles: A

1119 Review of EsxA in Mycobacterium-Host Interaction. Cells 10. 10.3390/cells10071645.

1120 Barker, L.P., George, K.M., Falkow, S., and Small, P.L. (1997). Differential trafficking of

1121 live and dead Mycobacterium marinum organisms in macrophages. Infect Immun 65,

1122 1497-1504. 10.1128/iai.65.4.1497-1504.1997.

1123 Beckwith, K.S., Beckwith, M.S., Ullmann, S., Saetra, R.S., Kim, H., Marstad, A., Asberg, 1124 S.E., Strand, T.A., Haug, M., Niederweis, M., et al. (2020). Plasma membrane damage 
1125 causes NLRP3 activation and pyroptosis during Mycobacterium tuberculosis infection.

1126 Nature communications 11, 2270. 10.1038/s41467-020-16143-6.

1127 Behar, S.M., Divangahi, M., and Remold, H.G. (2010). Evasion of innate immunity by

1128 Mycobacterium tuberculosis: is death an exit strategy? Nat Rev Microbiol 8, 668-674.

$1129 \quad 10.1038 /$ nrmicro2387.

1130 Behr, M.A., Edelstein, P.H., and Ramakrishnan, L. (2018). Revisiting the timetable of

1131 tuberculosis. BMJ 362, k2738. 10.1136/bmj.k2738.

1132 Behr, M.A., Edelstein, P.H., and Ramakrishnan, L. (2019). Is Mycobacterium

1133 tuberculosis infection life long? BMJ 367, I5770. 10.1136/bmj.15770.

1134 Behr, M.A., Kaufmann, E., Duffin, J., Edelstein, P.H., and Ramakrishnan, L. (2021).

1135 Latent Tuberculosis: Two Centuries of Confusion. Am J Respir Crit Care Med 204, 142-

1136 148. 10.1164/rccm.202011-4239PP.

1137 Bendall, L.J., and Green, D.R. (2014). Autopsy of a cell. Leukemia 28, 1341-1343.

$1138 \quad$ 10.1038/leu.2014.17.

1139 Benjamin, D., Colombi, M., Moroni, C., and Hall, M.N. (2011). Rapamycin passes the

1140 torch: a new generation of mTOR inhibitors. Nat Rev Drug Discov 10, 868-880.

$1141 \quad 10.1038 / \mathrm{nrd3531.}$

1142 Bento, C.F., Renna, M., Ghislat, G., Puri, C., Ashkenazi, A., Vicinanza, M., Menzies,

1143 F.M., and Rubinsztein, D.C. (2016). Mammalian Autophagy: How Does It Work? Annu

1144 Rev Biochem 85, 685-713. 10.1146/annurev-biochem-060815-014556.

1145 Berg, R.D., Levitte, S., O'Sullivan, M.P., O'Leary, S.M., Cambier, C.J., Cameron, J.,

1146 Takaki, K.K., Moens, C.B., Tobin, D.M., Keane, J., and Ramakrishnan, L. (2016). 
1147 Lysosomal Disorders Drive Susceptibility to Tuberculosis by Compromising

1148 Macrophage Migration. Cell 165, 139-152. 10.1016/j.cell.2016.02.034.

1149 Berthet, F.X., Lagranderie, M., Gounon, P., Laurent-Winter, C., Ensergueix, D.,

1150 Chavarot, P., Thouron, F., Maranghi, E., Pelicic, V., Portnoi, D., et al. (1998).

1151 Attenuation of virulence by disruption of the Mycobacterium tuberculosis erp gene.

1152 Science 282, 759-762. 10.1126/science.282.5389.759.

1153 Braverman, J., Sogi, K.M., Benjamin, D., Nomura, D.K., and Stanley, S.A. (2016). HIF-

1154 1alpha Is an Essential Mediator of IFN-gamma-Dependent Immunity to Mycobacterium

1155 tuberculosis. J Immunol 197, 1287-1297. 10.4049/jimmunol.1600266.

1156 Brodin, P., de Jonge, M.I., Majlessi, L., Leclerc, C., Nilges, M., Cole, S.T., and Brosch,

1157 R. (2005). Functional analysis of early secreted antigenic target-6, the dominant T-cell

1158 antigen of Mycobacterium tuberculosis, reveals key residues involved in secretion,

1159 complex formation, virulence, and immunogenicity. J Biol Chem 280, 33953-33959.

1160 10.1074/jbc.M503515200.

1161 Cambier, C.J., Banik, S.M., Buonomo, J.A., and Bertozzi, C.R. (2020). Spreading of a

1162 mycobacterial cell-surface lipid into host epithelial membranes promotes infectivity. Elife

1163 9. $10.7554 /$ eLife.60648.

1164 Cambier, C.J., Falkow, S., and Ramakrishnan, L. (2014a). Host evasion and

1165 exploitation schemes of Mycobacterium tuberculosis. Cell 159, 1497-1509.

1166 10.1016/j.cell.2014.11.024.

1167 Cambier, C.J., Takaki, K.K., Larson, R.P., Hernandez, R.E., Tobin, D.M., Urdahl, K.B.,

1168 Cosma, C.L., and Ramakrishnan, L. (2014b). Mycobacteria manipulate macrophage 
1169 recruitment through coordinated use of membrane lipids. Nature 505, 218-222.

$1170 \quad 10.1038 /$ nature12799.

1171 Canetti, G., Sutherland, I., and Svandova, E. (1972). Endogenous reactivation and

1172 exogenous reinfection: their relative importance with regard to the development of non-

1173 primary tuberculosis. Bull Int Union Tuberc 47, 116-134.

1174 Champion, M.M., Williams, E.A., Pinapati, R.S., and Champion, P.A. (2014). Correlation

1175 of phenotypic profiles using targeted proteomics identifies mycobacterial esx-1

1176 substrates. J Proteome Res 13, 5151-5164. 10.1021/pr500484w.

1177 Chen, M., Divangahi, M., Gan, H., Shin, D.S., Hong, S., Lee, D.M., Serhan, C.N., Behar,

1178 S.M., and Remold, H.G. (2008). Lipid mediators in innate immunity against tuberculosis:

1179 opposing roles of PGE2 and LXA4 in the induction of macrophage death. J Exp Med

1180 205, 2791-2801. jem.20080767 [pii]

1181 10.1084/jem.20080767.

1182 Clay, H., Volkman, H.E., and Ramakrishnan, L. (2008). Tumor necrosis factor signaling

1183 mediates resistance to mycobacteria by inhibiting bacterial growth and macrophage

1184 death. Immunity 29, 283-294.

1185 Clemens, D.L., and Horwitz, M.A. (1995). Characterization of the Mycobacterium

1186 tuberculosis phagosome and evidence that phagosomal maturation is inhibited. J Exp

1187 Med 181, 257-270. 10.1084/jem.181.1.257.

1188 Conrad, W.H., Osman, M.M., Shanahan, J.K., Chu, F., Takaki, K.K., Cameron, J.,

1189 Hopkinson-Woolley, D., Brosch, R., and Ramakrishnan, L. (2017). Mycobacterial ESX-1

1190 secretion system mediates host cell lysis through bacterium contact-dependent gross 
1191 membrane disruptions. Proc Natl Acad Sci U S A 114, 1371-1376.

1192 10.1073/pnas.1620133114.

1193 Cosma, C.L., Klein, K., Kim, R., Beery, D., and Ramakrishnan, L. (2006).

1194 Mycobacterium marinum Erp is a virulence determinant required for cell wall integrity

1195 and intracellular survival. Infect Immun 74, 3125-3133. 74/6/3125 [pii]

1196 10.1128/IAI.02061-05.

1197 Cosma, C.L., Sherman, D.R., and Ramakrishnan, L. (2003). The secret lives of the

1198 pathogenic mycobacteria. Annu Rev Microbiol 57, 641-676.

1199 Cumming, B.M., Addicott, K.W., Adamson, J.H., and Steyn, A.J. (2018). Mycobacterium

1200 tuberculosis induces decelerated bioenergetic metabolism in human macrophages. Elife

$1201 \quad 7.10 .7554 /$ eLife.39169.

1202 Cunningham, J.T., Rodgers, J.T., Arlow, D.H., Vazquez, F., Mootha, V.K., and

1203 Puigserver, P. (2007). mTOR controls mitochondrial oxidative function through a YY1-

1204 PGC-1alpha transcriptional complex. Nature 450, 736-740. 10.1038/nature06322.

1205 Davis, J.M., Clay, H., Lewis, J.L., Ghori, N., Herbomel, P., and Ramakrishnan, L.

1206 (2002). Real-time visualization of mycobacterium-macrophage interactions leading to

1207 initiation of granuloma formation in zebrafish embryos. Immunity $17,693-702$.

1208 Davis, J.M., and Ramakrishnan, L. (2009). The role of the granuloma in expansion and

1209 dissemination of early tuberculous infection. Cell 136, 37-49.

1210 Demy, D.L., Ranta, Z., Giorgi, J.M., Gonzalez, M., Herbomel, P., and Kissa, K. (2013).

1211 Generating parabiotic zebrafish embryos for cell migration and homing studies. Nature

1212 methods 10, 256-258. 10.1038/nmeth.2362. 
1213 Deng, W., Yang, J., Lin, X., Shin, J., Gao, J., and Zhong, X.P. (2017). Essential Role of

1214 mTORC1 in Self-Renewal of Murine Alveolar Macrophages. J Immunol 198, 492-504.

1215 10.4049/jimmunol.1501845.

1216 Divangahi, M., Chen, M., Gan, H., Desjardins, D., Hickman, T.T., Lee, D.M., Fortune,

1217 S., Behar, S.M., and Remold, H.G. (2009). Mycobacterium tuberculosis evades

1218 macrophage defenses by inhibiting plasma membrane repair. Nat Immunol 10, 899-906.

$1219 \quad 10.1038 /$ ni. 1758.

1220 Duvel, K., Yecies, J.L., Menon, S., Raman, P., Lipovsky, A.I., Souza, A.L., Triantafellow,

1221 E., Ma, Q., Gorski, R., Cleaver, S., et al. (2010). Activation of a metabolic gene

1222 regulatory network downstream of mTOR complex 1 . Mol Cell 39, 171-183.

$1223 \quad 10.1016 /$ j.molcel.2010.06.022.

1224 Edwards-Hicks, J., Mitterer, M., Pearce, E.L., and Buescher, J.M. (2020). Metabolic

1225 Dynamics of In Vitro CD8+ T Cell Activation. Metabolites 11. 10.3390/metabo11010012.

1226 Ehrt, S., Rhee, K., and Schnappinger, D. (2015). Mycobacterial genes essential for the

1227 pathogen's survival in the host. Immunol Rev 264, 319-326. 10.1111/imr.12256.

1228 Feldman, W.H., and Baggenstoss, A.H. (1938). The reisdual infectivity of the primary

1229 complex of tuberculosis. Am. J. Pathol. 14, 473-490.

1230 Fijalkowska-Morawska, J.B., Jagodzinska, M., and Nowicki, M. (2011). Pulmonary

1231 embolism and reactivation of tuberculosis during everolimus therapy in a kidney

1232 transplant recipient. Ann Transplant 16, 107-110. 10.12659/aot.882226.

1233 Fine-Coulson, K., Giguere, S., Quinn, F.D., and Reaves, B.J. (2015). Infection of A549

1234 human type II epithelial cells with Mycobacterium tuberculosis induces changes in 
1235 mitochondrial morphology, distribution and mass that are dependent on the early

1236 secreted antigen, ESAT-6. Microbes Infect 17, 689-697. 10.1016/j.micinf.2015.06.003.

1237 Fortune, S.M., Jaeger, A., Sarracino, D.A., Chase, M.R., Sassetti, C.M., Sherman, D.R.,

1238 Bloom, B.R., and Rubin, E.J. (2005). Mutually dependent secretion of proteins required

1239 for mycobacterial virulence. Proc Natl Acad Sci U S A 102, 10676-10681.

$1240 \quad 10.1073 /$ pnas.0504922102.

1241 Galluzzi, L., Bravo-San Pedro, J.M., Kepp, O., and Kroemer, G. (2016). Regulated cell

1242 death and adaptive stress responses. Cell Mol Life Sci 73, 2405-2410. 10.1007/s00018-

1243 016-2209-y.

1244 Garcia, C.A., and Wu, S. (2016). Attributable Risk of Infection to mTOR Inhibitors

1245 Everolimus and Temsirolimus in the Treatment of Cancer. Cancer Invest 34, 521-530.

$1246 \quad 10.1080 / 07357907.2016 .1242009$.

1247 Garritano, S., Gemignani, F., Voegele, C., Nguyen-Dumont, T., Le Calvez-Kelm, F., De

1248 Silva, D., Lesueur, F., Landi, S., and Tavtigian, S.V. (2009). Determining the

1249 effectiveness of High Resolution Melting analysis for SNP genotyping and mutation

1250 scanning at the TP53 locus. BMC Genet 10, 5. 10.1186/1471-2156-10-5.

1251 Gleeson, L.E., Sheedy, F.J., Palsson-McDermott, E.M., Triglia, D., O'Leary, S.M.,

1252 O'Sullivan, M.P., O'Neill, L.A., and Keane, J. (2016). Cutting Edge: Mycobacterium

1253 tuberculosis Induces Aerobic Glycolysis in Human Alveolar Macrophages That Is

1254 Required for Control of Intracellular Bacillary Replication. J Immunol 196, 2444-2449.

1255 10.4049/jimmunol.1501612. 
1256 Gonzalez, A., Hall, M.N., Lin, S.C., and Hardie, D.G. (2020). AMPK and TOR: The Yin

1257 and Yang of Cellular Nutrient Sensing and Growth Control. Cell Metab 31, 472-492.

$1258 \quad$ 10.1016/j.cmet.2020.01.015.

1259 Green, D.R., and Levine, B. (2014). To be or not to be? How selective autophagy and

1260 cell death govern cell fate. Cell 157, 65-75. 10.1016/j.cell.2014.02.049.

1261 Groschel, M.I., Sayes, F., Simeone, R., Majlessi, L., and Brosch, R. (2016). ESX

1262 secretion systems: mycobacterial evolution to counter host immunity. Nat Rev Microbiol

1263 14, 677-691. 10.1038/nrmicro.2016.131.

1264 Guinn, K.M., Hickey, M.J., Mathur, S.K., Zakel, K.L., Grotzke, J.E., Lewinsohn, D.M.,

1265 Smith, S., and Sherman, D.R. (2004). Individual RD1-region genes are required for

1266 export of ESAT-6/CFP-10 and for virulence of Mycobacterium tuberculosis. Mol

1267 Microbiol 51, 359-370. 10.1046/j.1365-2958.2003.03844.x.

1268 Halestrap, A.P. (1975). The mitochondrial pyruvate carrier. Kinetics and specificity for

1269 substrates and inhibitors. The Biochemical journal 148, 85-96. 10.1042/bj1480085.

1270 Harris, J., Hope, J.C., and Keane, J. (2008). Tumor necrosis factor blockers influence

1271 macrophage responses to Mycobacterium tuberculosis. J Infect Dis 198, 1842-1850.

$1272 \quad 10.1086 / 593174$.

1273 He, C., Bartholomew, C.R., Zhou, W., and Klionsky, D.J. (2009). Assaying autophagic

1274 activity in transgenic GFP-Lc3 and GFP-Gabarap zebrafish embryos. Autophagy 5, 520-

1275 526. 10.4161/auto.5.4.7768.

1276 He, C., Holme, J., and Anthony, J. (2014). SNP genotyping: the KASP assay. Methods

1277 Mol Biol 1145, 75-86. 10.1007/978-1-4939-0446-4_7. 
1278 Hinman, A.E., Jani, C., Pringle, S.C., Zhang, W.R., Jain, N., Martinot, A.J., and Barczak,

1279 A.K. (2021). Mycobacterium tuberculosis canonical virulence factors interfere with a late

1280 component of the TLR2 response. Elife 10.10.7554/eLife.73984.

1281 Holscher, C., Reiling, N., Schaible, U.E., Holscher, A., Bathmann, C., Korbel, D., Lenz,

1282 I., Sonntag, T., Kroger, S., Akira, S., et al. (2008). Containment of aerogenic

1283 Mycobacterium tuberculosis infection in mice does not require MyD88 adaptor function

1284 for TLR2, -4 and -9. Eur J Immunol 38, 680-694. 10.1002/eji.200736458.

1285 Hopfner, K.P., and Hornung, V. (2020). Molecular mechanisms and cellular functions of

1286 cGAS-STING signalling. Nat Rev Mol Cell Biol 21, 501-521. 10.1038/s41580-020-0244-

$1287 x$

1288 Hosoi, H., Dilling, M.B., Shikata, T., Liu, L.N., Shu, L., Ashmun, R.A., Germain, G.S.,

1289 Abraham, R.T., and Houghton, P.J. (1999). Rapamycin causes poorly reversible

1290 inhibition of mTOR and induces p53-independent apoptosis in human

1291 rhabdomyosarcoma cells. Cancer Res 59, 886-894.

1292 Huang, L., Nazarova, E.V., Tan, S., Liu, Y., and Russell, D.G. (2018). Growth of

1293 Mycobacterium tuberculosis in vivo segregates with host macrophage metabolism and

1294 ontogeny. J Exp Med 215, 1135-1152. 10.1084/jem.20172020.

1295 Jamwal, S., Midha, M.K., Verma, H.N., Basu, A., Rao, K.V., and Manivel, V. (2013).

1296 Characterizing virulence-specific perturbations in the mitochondrial function of

1297 macrophages infected with Mycobacterium tuberculosis. Scientific reports 3, 1328.

$1298 \quad 10.1038 /$ srep01328.

1299 Jeon, S.Y., Yhim, H.Y., Lee, N.R., Song, E.K., Kwak, J.Y., and Yim, C.Y. (2017).

1300 Everolimus-induced activation of latent Mycobacterium tuberculosis infection in a patient 
1301 with metastatic renal cell carcinoma. Korean J Intern Med 32, 365-368.

$1302 \quad$ 10.3904/kjim.2015.121.

1303 Karmaus, P.W.F., Herrada, A.A., Guy, C., Neale, G., Dhungana, Y., Long, L., Vogel, P.,

1304 Avila, J., Clish, C.B., and Chi, H. (2017). Critical roles of mTORC1 signaling and

1305 metabolic reprogramming for M-CSF-mediated myelopoiesis. J Exp Med 214, 2629-

1306 2647. 10.1084/jem.20161855.

1307 Kettleborough, R.N., Busch-Nentwich, E.M., Harvey, S.A., Dooley, C.M., de Bruijn, E.,

1308 van Eeden, F., Sealy, I., White, R.J., Herd, C., Nijman, I.J., et al. (2013). A systematic

1309 genome-wide analysis of zebrafish protein-coding gene function. Nature 496, 494-497.

$1310 \quad 10.1038 /$ nature11992.

1311 Khattak, S., Murawala, P., Andreas, H., Kappert, V., Schuez, M., Sandoval-Guzman, T.,

1312 Crawford, K., and Tanaka, E.M. (2014). Optimized axolotl (Ambystoma mexicanum)

1313 husbandry, breeding, metamorphosis, transgenesis and tamoxifen-mediated

1314 recombination. Nature protocols 9, 529-540. 10.1038/nprot.2014.040.

1315 Kiritsy, M.C., McCann, K., Mott, D., Holland, S.M., Behar, S.M., Sassetti, C.M., and

1316 Olive, A.J. (2021). Mitochondrial respiration contributes to the interferon gamma

1317 response in antigen-presenting cells. Elife 10. 10.7554/eLife.65109.

1318 Kozik, P., Gros, M., Itzhak, D.N., Joannas, L., Heurtebise-Chretien, S., Krawczyk, P.A.,

1319 Rodriguez-Silvestre, P., Alloatti, A., Magalhaes, J.G., Del Nery, E., et al. (2020). Small

1320 Molecule Enhancers of Endosome-to-Cytosol Import Augment Anti-tumor Immunity. Cell

1321 reports 32, 107905. 10.1016/j.celrep.2020.107905.

1322 Lachmandas, E., Beigier-Bompadre, M., Cheng, S.C., Kumar, V., van Laarhoven, A.,

1323 Wang, X., Ammerdorffer, A., Boutens, L., de Jong, D., Kanneganti, T.D., et al. (2016). 
1324 Rewiring cellular metabolism via the AKT/mTOR pathway contributes to host defence

1325 against Mycobacterium tuberculosis in human and murine cells. Eur J Immunol 46,

1326 2574-2586. 10.1002/eji.201546259.

1327 Lee, J., Repasy, T., Papavinasasundaram, K., Sassetti, C., and Kornfeld, H. (2011).

1328 Mycobacterium tuberculosis induces an atypical cell death mode to escape from

1329 infected macrophages. PLoS One 6, e18367. 10.1371/journal.pone.0018367.

1330 Lee, P.Y., Sykes, D.B., Ameri, S., Kalaitzidis, D., Charles, J.F., Nelson-Maney, N., Wei,

1331 K., Cunin, P., Morris, A., Cardona, A.E., et al. (2017). The metabolic regulator mTORC1

1332 controls terminal myeloid differentiation. Science immunology 2.

1333 10.1126/sciimmunol.aam6641.

1334 Lerner, T.R., Queval, C.J., Fearns, A., Repnik, U., Griffiths, G., and Gutierrez, M.G.

1335 (2018). Phthiocerol dimycocerosates promote access to the cytosol and intracellular

1336 burden of Mycobacterium tuberculosis in lymphatic endothelial cells. BMC Biol 16, 1.

1337 10.1186/s12915-017-0471-6.

1338 Levitte, S., Adams, K.N., Berg, R.D., Cosma, C.L., Urdahl, K.B., and Ramakrishnan, L.

1339 (2016). Mycobacterial Acid Tolerance Enables Phagolysosomal Survival and

1340 Establishment of Tuberculous Infection In Vivo. Cell Host Microbe 20, 250-258.

$1341 \quad$ 10.1016/j.chom.2016.07.007.

1342 Lienard, J., Nobs, E., Lovins, V., Movert, E., Valfridsson, C., and Carlsson, F. (2020).

1343 The Mycobacterium marinum ESX-1 system mediates phagosomal permeabilization

1344 and type I interferon production via separable mechanisms. Proc Natl Acad Sci U S A

1345 117, 1160-1166. 10.1073/pnas.1911646117. 
1346 Liu, G.Y., and Sabatini, D.M. (2020). mTOR at the nexus of nutrition, growth, ageing

1347 and disease. Nat Rev Mol Cell Biol 21, 183-203. 10.1038/s41580-019-0199-y.

1348 Manzanillo, P.S., Shiloh, M.U., Portnoy, D.A., and Cox, J.S. (2012). Mycobacterium

1349 tuberculosis activates the DNA-dependent cytosolic surveillance pathway within

1350 macrophages. Cell Host Microbe 11, 469-480. 10.1016/j.chom.2012.03.007.

1351 Mayer-Barber, K.D., Andrade, B.B., Oland, S.D., Amaral, E.P., Barber, D.L., Gonzales,

1352 J., Derrick, S.C., Shi, R., Kumar, N.P., Wei, W., et al. (2014). Host-directed therapy of

1353 tuberculosis based on interleukin-1 and type I interferon crosstalk. Nature 511, 99-103.

1354 10.1038/nature13489.

1355 Mayer-Barber, K.D., Barber, D.L., Shenderov, K., White, S.D., Wilson, M.S., Cheever,

1356 A., Kugler, D., Hieny, S., Caspar, P., Nunez, G., et al. (2010). Caspase-1 independent

1357 IL-1beta production is critical for host resistance to mycobacterium tuberculosis and

1358 does not require TLR signaling in vivo. J Immunol 184, 3326-3330.

1359 10.4049/jimmunol.0904189.

1360 Mercalli, A., Sordi, V., Ponzoni, M., Maffi, P., De Taddeo, F., Gatti, G., Servida, P.,

1361 Bernardi, M., Bellio, L., Bertuzzi, F., et al. (2006). Rapamycin induces a caspase-

1362 independent cell death in human monocytes. Am J Transplant 6, 1331-1341.

1363 10.1111/j.1600-6143.2006.01332.x.

1364 Mohareer, K., Medikonda, J., Vadankula, G.R., and Banerjee, S. (2020). Mycobacterial

1365 Control of Host Mitochondria: Bioenergetic and Metabolic Changes Shaping Cell Fate 1366 and Infection Outcome. Front Cell Infect Microbiol 10, 457. 10.3389/fcimb.2020.00457. 
1367 Moreira-Teixeira, L., Mayer-Barber, K., Sher, A., and O'Garra, A. (2018). Type I

1368 interferons in tuberculosis: Foe and occasionally friend. J Exp Med 215, 1273-1285.

$1369 \quad 10.1084 /$ jem.20180325.

1370 Morita, M., Gravel, S.P., Chenard, V., Sikstrom, K., Zheng, L., Alain, T., Gandin, V.,

1371 Avizonis, D., Arguello, M., Zakaria, C., et al. (2013). mTORC1 controls mitochondrial

1372 activity and biogenesis through 4E-BP-dependent translational regulation. Cell Metab

1373 18, 698-711. 10.1016/j.cmet.2013.10.001.

1374 Morita, M., Prudent, J., Basu, K., Goyon, V., Katsumura, S., Hulea, L., Pearl, D.,

1375 Siddiqui, N., Strack, S., McGuirk, S., et al. (2017). mTOR Controls Mitochondrial

1376 Dynamics and Cell Survival via MTFP1. Mol Cell 67, 922-935 e925.

$1377 \quad$ 10.1016/j.molcel.2017.08.013.

1378 Mosimann, C., Kaufman, C.K., Li, P., Pugach, E.K., Tamplin, O.J., and Zon, L.I. (2011).

1379 Ubiquitous transgene expression and Cre-based recombination driven by the ubiquitin

1380 promoter in zebrafish. Development 138, 169-177. 10.1242/dev.059345.

1381 Mouton, J.M., Heunis, T., Dippenaar, A., Gallant, J.L., Kleynhans, L., and Sampson,

1382 S.L. (2019). Comprehensive Characterization of the Attenuated Double Auxotroph

1383 Mycobacterium tuberculosisDeltaleuDDeltapanCD as an Alternative to H37Rv. Front

1384 Microbiol 10, 1922. 10.3389/fmicb.2019.01922.

1385 Murphy, M.P. (2009). How mitochondria produce reactive oxygen species. The

1386 Biochemical journal 417, 1-13. 10.1042/BJ20081386.

1387 Muthukkumar, S., Ramesh, T.M., and Bondada, S. (1995). Rapamycin, a potent

1388 immunosuppressive drug, causes programmed cell death in B lymphoma cells.

1389 Transplantation 60, 264-270. 10.1097/00007890-199508000-00010. 
1390 O'Neill, L.A., and Pearce, E.J. (2016). Immunometabolism governs dendritic cell and

1391 macrophage function. J Exp Med 213, 15-23. 10.1084/jem.20151570.

1392 Olson, G.S., Murray, T.A., Jahn, A.N., Mai, D., Diercks, A.H., Gold, E.S., and Aderem,

1393 A. (2021). Type I interferon decreases macrophage energy metabolism during

1394 mycobacterial infection. Cell reports 35, 109195. 10.1016/j.celrep.2021.109195.

1395 Ong, C.W., Elkington, P.T., and Friedland, J.S. (2014). Tuberculosis, pulmonary

1396 cavitation, and matrix metalloproteinases. Am J Respir Crit Care Med 190, 9-18.

1397 10.1164/rccm.201311-2106PP.

1398 Opie, E.L., and Aronson, J.D. (1927). Tubercle bacilli in latent tuberculous lesions and

1399 in lung tissue without tuberculous lesions. Arch Pathol Lab Med 4, 1-21.

1400 Osman, M.M., Pagan, A.J., Shanahan, J.K., and Ramakrishnan, L. (2020).

1401 Mycobacterium marinum phthiocerol dimycocerosates enhance macrophage

1402 phagosomal permeabilization and membrane damage. PLoS One 15, e0233252.

$1403 \quad 10.1371 /$ journal.pone.0233252.

1404 Osman, M.M., Shanahan, J.K., Chu, F., Takaki, K.K., Pinckert, M.L., Pagán, A.J.,

1405 Brosch, R., Conrad, W.H., and Ramakrishnan, L. (2022). The C terminus of the

1406 mycobacterium ESX-1 secretion system substrate ESAT-6 is required for phagosomal

1407 membrane damage and virulence. Proc Natl Acad Sci U S A. (in press)

1408 https://www.biorxiv.org/content/10.1101/2022.01.14.476355v1.

1409 Pagan, A.J., and Ramakrishnan, L. (2014). Immunity and Immunopathology in the

1410 Tuberculous Granuloma. Cold Spring Harbor perspectives in medicine 5.

$1411 \quad$ 10.1101/cshperspect.a018499. 
1412 Pagan, A.J., and Ramakrishnan, L. (2018). The Formation and Function of Granulomas.

1413 Annu Rev Immunol 36, 639-665. 10.1146/annurev-immunol-032712-100022.

1414 Pagan, A.J., Yang, C.T., Cameron, J., Swaim, L.E., Ellett, F., Lieschke, G.J., and

1415 Ramakrishnan, L. (2015). Myeloid Growth Factors Promote Resistance to Mycobacterial

1416 Infection by Curtailing Granuloma Necrosis through Macrophage Replenishment. Cell

1417 Host Microbe 18, 15-26. 10.1016/j.chom.2015.06.008.

1418 Pajak, B., Siwiak, E., Soltyka, M., Priebe, A., Zielinski, R., Fokt, I., Ziemniak, M.,

1419 Jaskiewicz, A., Borowski, R., Domoradzki, T., and Priebe, W. (2019). 2-Deoxy-d-

1420 Glucose and Its Analogs: From Diagnostic to Therapeutic Agents. Int J Mol Sci 21.

$1421 \quad 10.3390 /$ ijms21010234.

1422 Pang, Z., Chong, J., Zhou, G., de Lima Morais, D.A., Chang, L., Barrette, M., Gauthier,

1423 C., Jacques, P.E., Li, S., and Xia, J. (2021). MetaboAnalyst 5.0: narrowing the gap

1424 between raw spectra and functional insights. Nucleic Acids Res 49, W388-W396.

$142510.1093 /$ nar/gkab382.

1426 Patra, K.C., and Hay, N. (2014). The pentose phosphate pathway and cancer. Trends

1427 Biochem Sci 39, 347-354. 10.1016/j.tibs.2014.06.005.

1428 Paulson, T. (2013). Epidemiology: A mortal foe. Nature 502, S2-3. 10.1038/502S2a.

1429 Pernas, L. (2021). Cellular metabolism in the defense against microbes. J Cell Sci 134.

$1430 \quad 10.1242 /$ jcs.252023.

1431 Powell, J.D., Pollizzi, K.N., Heikamp, E.B., and Horton, M.R. (2012). Regulation of

1432 immune responses by mTOR. Annu Rev Immunol 30, 39-68. 10.1146/annurev-

1433 immunol-020711-075024. 
1434 Quigley, J., Hughitt, V.K., Velikovsky, C.A., Mariuzza, R.A., El-Sayed, N.M., and Briken, 1435 V. (2017). The Cell Wall Lipid PDIM Contributes to Phagosomal Escape and Host Cell

1436 Exit of Mycobacterium tuberculosis. mBio 8. 10.1128/mBio.00148-17.

1437 Ramakrishnan, L. (2012). Revisiting the role of the granuloma in tuberculosis. Nat Rev

1438 Immunol 12, 352-366. 10.1038/nri3211.

1439 Ramakrishnan, L. (2013). The zebrafish guide to tuberculosis immunity and treatment.

1440 Cold Spring Harb Symp Quant Biol 78, 179-192. 10.1101/sqb.2013.78.023283.

1441 Ramakrishnan, L. (2020). Mycobacterium tuberculosis pathogenicity viewed through the

1442 lens of molecular Koch's postulates. Curr Opin Microbiol 54, 103-110.

1443 10.1016/j.mib.2020.01.011.

1444 Rambold, A.S., and Pearce, E.L. (2018). Mitochondrial Dynamics at the Interface of

1445 Immune Cell Metabolism and Function. Trends Immunol 39, 6-18.

1446 10.1016/j.it.2017.08.006.

1447 Randow, F., and Youle, R.J. (2014). Self and nonself: how autophagy targets

1448 mitochondria and bacteria. Cell Host Microbe 15, 403-411.

$1449 \quad$ 10.1016/j.chom.2014.03.012.

1450 Roca, F.J., and Ramakrishnan, L. (2013). TNF dually mediates resistance and

1451 susceptibility to mycobacteria via mitochondrial reactive oxygen species. Cell 153, 521-

1452 534. 10.1016/j.cell.2013.03.022.

1453 Roca, F.J., Whitworth, L.J., Redmond, S., Jones, A.A., and Ramakrishnan, L. (2019).

1454 TNF Induces Pathogenic Programmed Macrophage Necrosis in Tuberculosis through a

1455 Mitochondrial-Lysosomal-Endoplasmic Reticulum Circuit. Cell 178, 1344-1361 e1311.

$1456 \quad 10.1016 / j . c e l l .2019 .08 .004$. 
1457 Roca, F.J., Whitworth, L.J., Prag, H.A., Murphy, M.P., and Ramakrishnan, L. (2022).

1458 TNF induces reverse electron transport through complex I to produce pathogenic

1459 mitochondrial reactive oxygen species and macrophage necrosis in tuberculosis.

1460 Submitted.

1461 Ruiz-Camps, I., and Aguilar-Company, J. (2021). Risk of infection associated with

1462 targeted therapies for solid organ and hematological malignancies. Ther Adv Infect Dis

1463 8, 2049936121989548. 10.1177/2049936121989548.

1464 Russell, D.G. (2007). Who puts the tubercle in tuberculosis? Nat Rev Microbiol 5, 39-47.

1465 Ryan, D.G., and O'Neill, L.A.J. (2020). Krebs Cycle Reborn in Macrophage

1466 Immunometabolism. Annu Rev Immunol 38, 289-313. 10.1146/annurev-immunol-

$1467 \quad 081619-104850$.

1468 Sampson, S.L., Dascher, C.C., Sambandamurthy, V.K., Russell, R.G., Jacobs, W.R.,

1469 Jr., Bloom, B.R., and Hondalus, M.K. (2004). Protection elicited by a double leucine and 1470 pantothenate auxotroph of Mycobacterium tuberculosis in guinea pigs. Infect Immun 72,

$1471 \quad 3031-3037.10 .1128 /$ IAI.72.5.3031-3037.2004.

1472 Sampson, S.L., Mansfield, K.G., Carville, A., Magee, D.M., Quitugua, T., Howerth, E.W.,

1473 Bloom, B.R., and Hondalus, M.K. (2011). Extended safety and efficacy studies of a live

1474 attenuated double leucine and pantothenate auxotroph of Mycobacterium tuberculosis

1475 as a vaccine candidate. Vaccine 29, 4839-4847. 10.1016/j.vaccine.2011.04.066.

1476 Schieke, S.M., Phillips, D., McCoy, J.P., Jr., Aponte, A.M., Shen, R.F., Balaban, R.S.,

1477 and Finkel, T. (2006). The mammalian target of rapamycin (mTOR) pathway regulates

1478 mitochondrial oxygen consumption and oxidative capacity. J Biol Chem 281, 27643-

1479 27652. 10.1074/jbc.M603536200. 
1480 Shi, L., Salamon, H., Eugenin, E.A., Pine, R., Cooper, A., and Gennaro, M.L. (2015).

1481 Infection with Mycobacterium tuberculosis induces the Warburg effect in mouse lungs.

1482 Scientific reports 5, 18176. 10.1038/srep18176.

1483 Simeone, R., Bobard, A., Lippmann, J., Bitter, W., Majlessi, L., Brosch, R., and Enninga,

1484 J. (2012). Phagosomal rupture by Mycobacterium tuberculosis results in toxicity and

1485 host cell death. PLoS Pathog 8, e1002507. 10.1371/journal.ppat.1002507.

1486 Simeone, R., Sayes, F., Lawaree, E., and Brosch, R. (2021). Breaching the

1487 phagosome, the case of the tuberculosis agent. Cell Microbiol 23, e13344.

$1488 \quad 10.1111 / \mathrm{cmi} .13344$.

1489 Sinclair, C., Bommakanti, G., Gardinassi, L., Loebbermann, J., Johnson, M.J.,

1490 Hakimpour, P., Hagan, T., Benitez, L., Todor, A., Machiah, D., et al. (2017). mTOR

1491 regulates metabolic adaptation of APCs in the lung and controls the outcome of allergic

1492 inflammation. Science 357, 1014-1021. 10.1126/science.aaj2155.

1493 Small, J.L., O'Donoghue, A.J., Boritsch, E.C., Tsodikov, O.V., Knudsen, G.M., Vandal,

1494 O., Craik, C.S., and Ehrt, S. (2013). Substrate specificity of MarP, a periplasmic

1495 protease required for resistance to acid and oxidative stress in Mycobacterium

1496 tuberculosis. J Biol Chem 288, 12489-12499. 10.1074/jbc.M113.456541.

1497 Srinivasan, L., Ahlbrand, S., and Briken, V. (2014). Interaction of Mycobacterium

1498 tuberculosis with host cell death pathways. Cold Spring Harbor perspectives in medicine

1499 4. 10.1101/cshperspect.a022459.

1500 Stanley, S.A., Johndrow, J.E., Manzanillo, P., and Cox, J.S. (2007). The Type I IFN

1501 response to infection with Mycobacterium tuberculosis requires ESX-1-mediated 
1502 secretion and contributes to pathogenesis. J Immunol 178, 3143-3152.

$1503 \quad 10.4049 /$ jimmunol.178.5.3143.

1504 Sun, J., Siroy, A., Lokareddy, R.K., Speer, A., Doornbos, K.S., Cingolani, G., and

1505 Niederweis, M. (2015). The tuberculosis necrotizing toxin kills macrophages by

1506 hydrolyzing NAD. Nat Struct Mol Biol 22, 672-678. 10.1038/nsmb.3064.

1507 Suster, M.L., Abe, G., Schouw, A., and Kawakami, K. (2011). Transposon-mediated

1508 BAC transgenesis in zebrafish. Nature protocols 6, 1998-2021. 10.1038/nprot.2011.416.

1509 Swaim, L.E., Connolly, L.E., Volkman, H.E., Humbert, O., Born, D.E., and

1510 Ramakrishnan, L. (2006). Mycobacterium marinum infection of adult zebrafish causes

1511 caseating granulomatous tuberculosis and is moderated by adaptive immunity. Infect

1512 Immun 74, 6108-6117.

1513 Takaki, K., Davis, J.M., Winglee, K., and Ramakrishnan, L. (2013). Evaluation of the

1514 pathogenesis and treatment of Mycobacterium marinum infection in zebrafish. Nature

1515 protocols 8, 1114-1124. 10.1038/nprot.2013.068.

1516 Terplan, K. (1951). [Pathogenesis of postprimary tuberculosis, in relation to chronic

1517 pulmonary tuberculosis (phtisis)]. Bibl Tuberc 5, 186-219.

1518 Thomas, E.D., Cruz, I.A., Hailey, D.W., and Raible, D.W. (2015). There and back again:

1519 development and regeneration of the zebrafish lateral line system. Wiley Interdiscip Rev

1520 Dev Biol 4, 1-16. 10.1002/wdev.160.

1521 Tobin, D.M., Roca, F.J., Oh, S.F., McFarland, R., Vickery, T.W., Ray, J.P., Ko, D.C.,

1522 Zou, Y., Bang, N.D., Chau, T.T., et al. (2012). Host genotype-specific therapies can

1523 optimize the inflammatory response to mycobacterial infections. Cell 148, 434-446.

$1524 \quad$ 10.1016/j.cell.2011.12.023. 
1525 Tobin, D.M., Vary, J.C., Jr., Ray, J.P., Walsh, G.S., Dunstan, S.J., Bang, N.D., Hagge,

1526 D.A., Khadge, S., King, M.C., Hawn, T.R., et al. (2010). The Ita4h locus modulates

1527 susceptibility to mycobacterial infection in zebrafish and humans. Cell 140, 717-730.

$1528 \quad$ 10.1016/j.cell.2010.02.013.

1529 Truett, G.E., Heeger, P., Mynatt, R.L., Truett, A.A., Walker, J.A., and Warman, M.L.

1530 (2000). Preparation of PCR-quality mouse genomic DNA with hot sodium hydroxide and

1531 tris (HotSHOT). BioTechniques 29, 52, 54. 10.2144/00291bm09.

1532 Tsai, M.K., Lee, C.Y., Hu, R.H., and Lee, P.H. (2007). Conversion to combined therapy

1533 with sirolimus and mycophenolate mofetil improved renal function in stable renal

1534 transplant recipients. J Formos Med Assoc 106, 372-379. 10.1016/S0929-

$1535 \quad 6646(09) 60322-3$.

1536 van Ham, T.J., Mapes, J., Kokel, D., and Peterson, R.T. (2010). Live imaging of

1537 apoptotic cells in zebrafish. FASEB J 24, 4336-4342. 10.1096/fj.10-161018.

1538 Vandal, O.H., Pierini, L.M., Schnappinger, D., Nathan, C.F., and Ehrt, S. (2008). A

1539 membrane protein preserves intrabacterial $\mathrm{pH}$ in intraphagosomal Mycobacterium

1540 tuberculosis. Nat Med 14, 849-854. 10.1038/nm.1795.

1541 Volkman, H.E., Clay, H., Beery, D., Chang, J.C., Sherman, D.R., and Ramakrishnan, L.

1542 (2004). Tuberculous granuloma formation is enhanced by a mycobacterium virulence

1543 determinant. PLoS Biol 2, e367.

1544 Walton, E.M., Cronan, M.R., Beerman, R.W., and Tobin, D.M. (2015). The Macrophage-

1545 Specific Promoter mfap4 Allows Live, Long-Term Analysis of Macrophage Behavior

1546 during Mycobacterial Infection in Zebrafish. PLoS One 10, e0138949.

1547 10.1371/journal.pone.0138949. 
1548 Weichhart, T., Hengstschlager, M., and Linke, M. (2015). Regulation of innate immune

1549 cell function by mTOR. Nat Rev Immunol 15, 599-614. 10.1038/nri3901.

1550 Whitworth, L., Coxon, J., van Laarhoven, A., Thuong, N.T.T., Dian, S., Alisjahbana, B.,

1551 Ganiem, A.R., van Crevel, R., Thwaites, G.E., Troll, M., et al. (2021a). A Bayesian

1552 analysis of the association between Leukotriene A4 Hydrolase genotype and survival in

1553 tuberculous meningitis. Elife 10.10.7554/eLife.61722.

1554 Whitworth, L.J., Troll, R., Pagan, A.J., Roca, F.J., Edelstein, P.H., Troll, M., Tobin, D.M.,

1555 Phu, N.H., Bang, N.D., Thwaites, G.E., et al. (2021b). Elevated cerebrospinal fluid

1556 cytokine levels in tuberculous meningitis predict survival in response to dexamethasone.

1557 Proc Natl Acad Sci U S A 118. 10.1073/pnas.2024852118.

1558 Wiens, K.E., and Ernst, J.D. (2016). The Mechanism for Type I Interferon Induction by

1559 Mycobacterium tuberculosis is Bacterial Strain-Dependent. PLoS Pathog 12, e1005809.

1560 10.1371/journal.ppat.1005809.

1561 Wills, J., Edwards-Hicks, J., and Finch, A.J. (2017). AssayR: A Simple Mass

1562 Spectrometry Software Tool for Targeted Metabolic and Stable Isotope Tracer

1563 Analyses. Anal Chem 89, 9616-9619. 10.1021/acs.analchem.7b02401.

1564 Wong, Y.C., Ysselstein, D., and Krainc, D. (2018). Mitochondria-lysosome contacts

1565 regulate mitochondrial fission via RAB7 GTP hydrolysis. Nature 554, 382-386.

$156610.1038 /$ nature25486.

1567 Zhang, L., Jiang, X., Pfau, D., Ling, Y., and Nathan, C.F. (2021). Type I interferon

1568 signaling mediates Mycobacterium tuberculosis-induced macrophage death. J Exp Med

1569 218. 10.1084/jem.20200887. 
1570 Zhang, Q., Wang, D., Jiang, G., Liu, W., Deng, Q., Li, X., Qian, W., Ouellet, H., and

1571 Sun, J. (2016). EsxA membrane-permeabilizing activity plays a key role in

1572 mycobacterial cytosolic translocation and virulence: effects of single-residue mutations

1573 at glutamine 5. Scientific reports 6, 32618. 10.1038/srep32618.

1574 
Figure 1

A

hbv
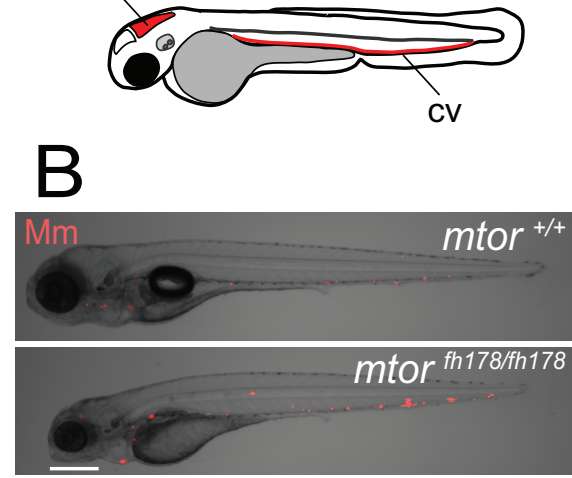

E

$\square$ not cording $\quad \mathrm{F}$ cording

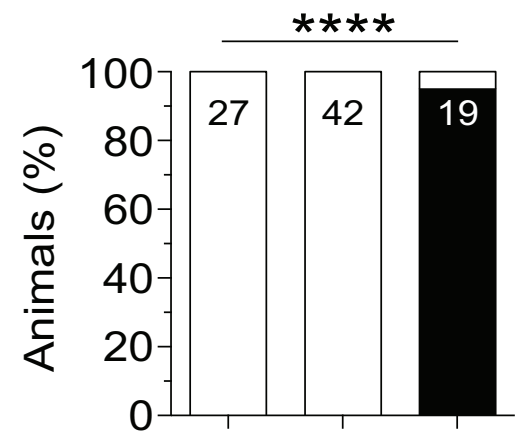

mtor: $x^{1 x}$

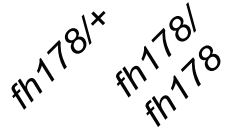

$\mathrm{H}$

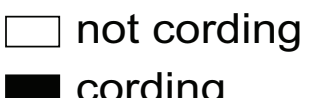

cording

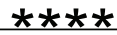

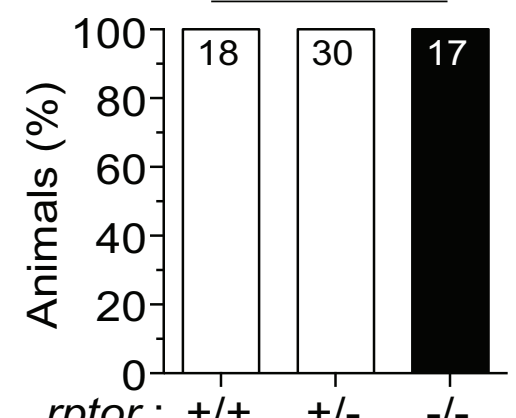

rptor : $+/+\quad+/-\quad-/-$

C

1

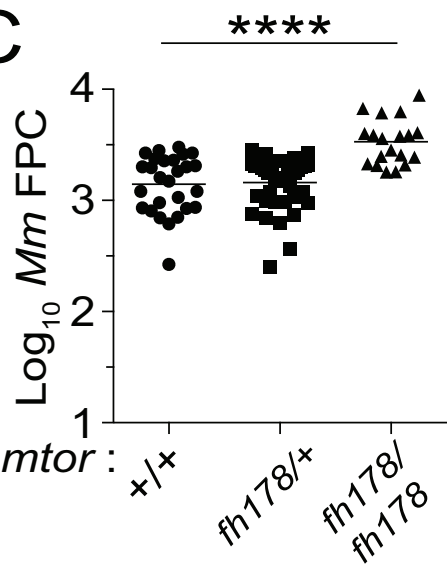

$\square$ not cording cording

$\star \star * *$

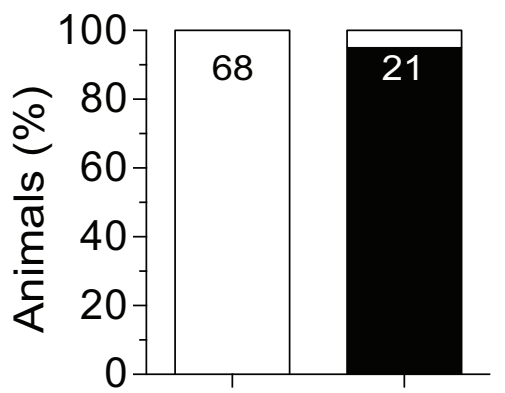

mtor: +/+ sa16755/ sa16755/+ sa16755

$\square$ not cording $J$ cording

$\mathrm{G}$

MФ $\quad m t^{f h 178 / / h 178}$ $\mathrm{Mm}$

МФ $\quad m t^{\text {fh178/fh178 }}$

mtor ${ }^{+/+}$
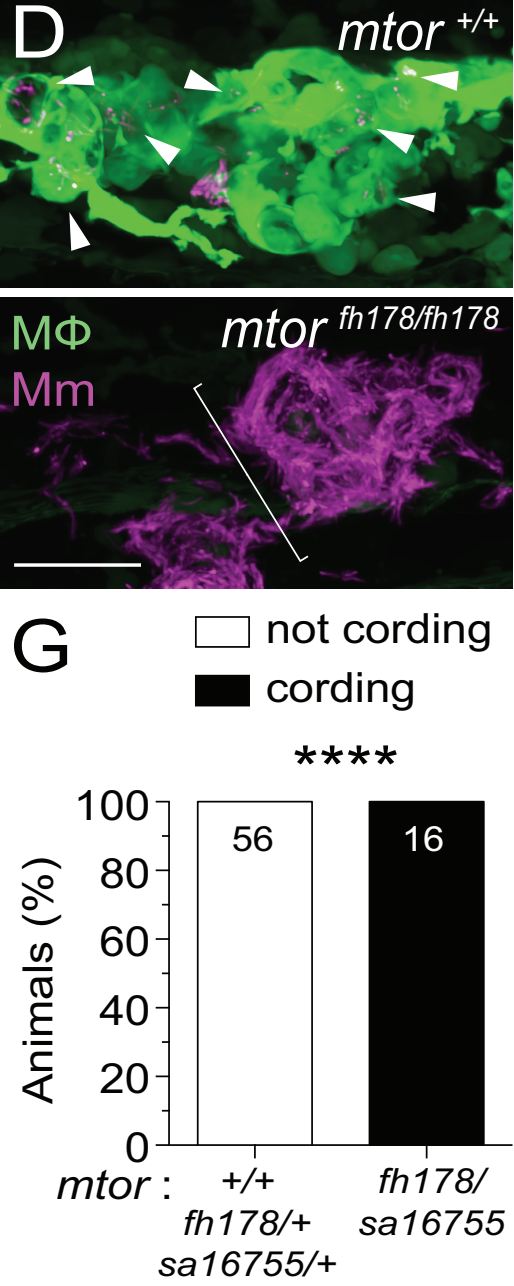

$\square$ not cording cording

****

$\star * \star *$
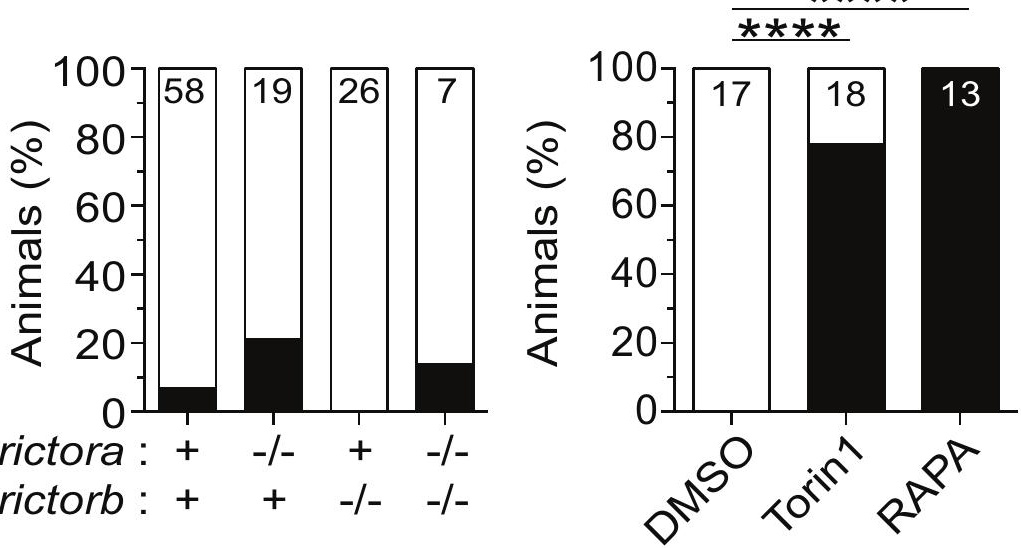

rictora : + -/- + -/-

rictorb : + + -/- -/- 
bioRxiv prepiptppi tres://Oorg/10.1101/2022.01.30.478369; this version posted January 30, 2022. The copyright holder for this preprint (which wat ndt review) is the author/funder, who has granted bioRxiv a license to display the preprint in perpetuity. It is made available under aCC-BY 4.0 International license.
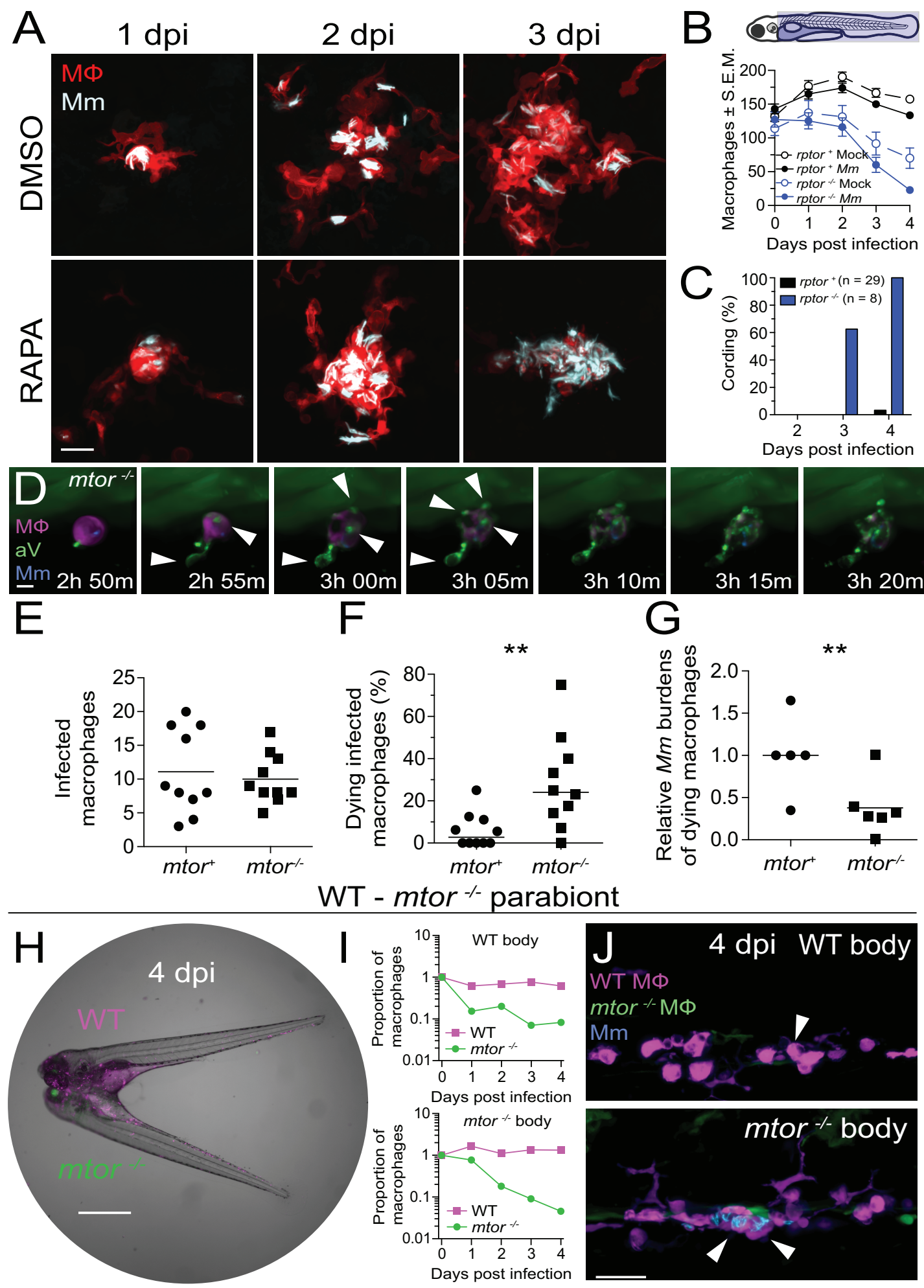

\section{WT - mtor ${ }^{+/}$parabiont}
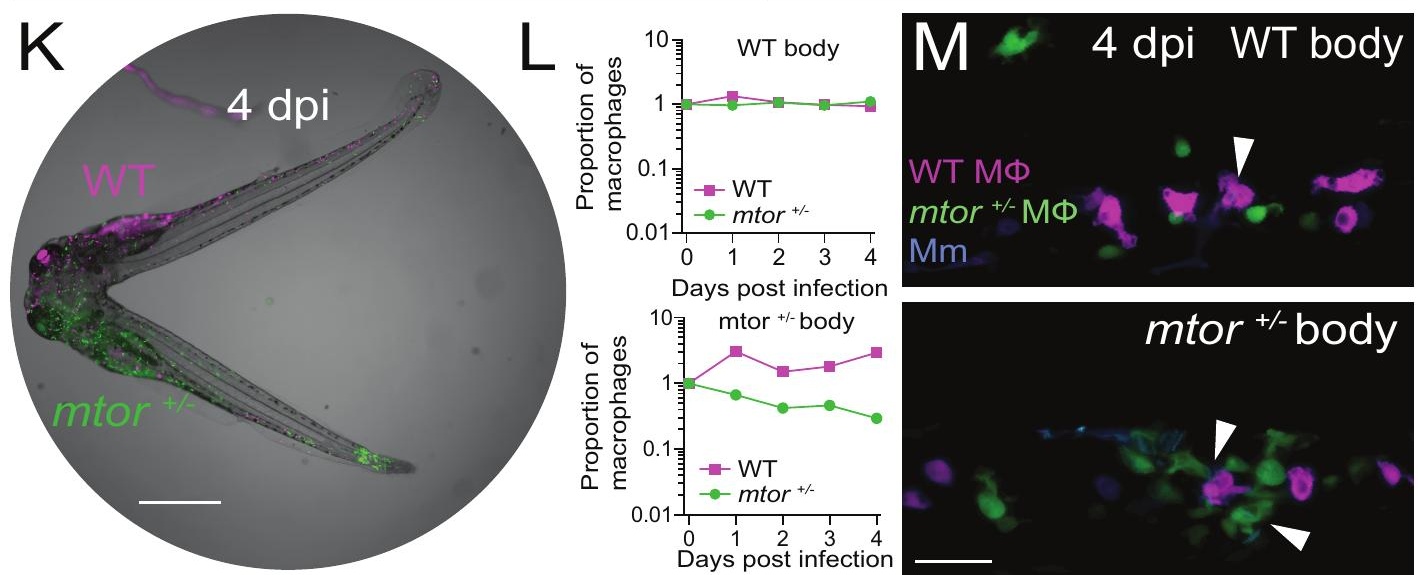
Figure 3
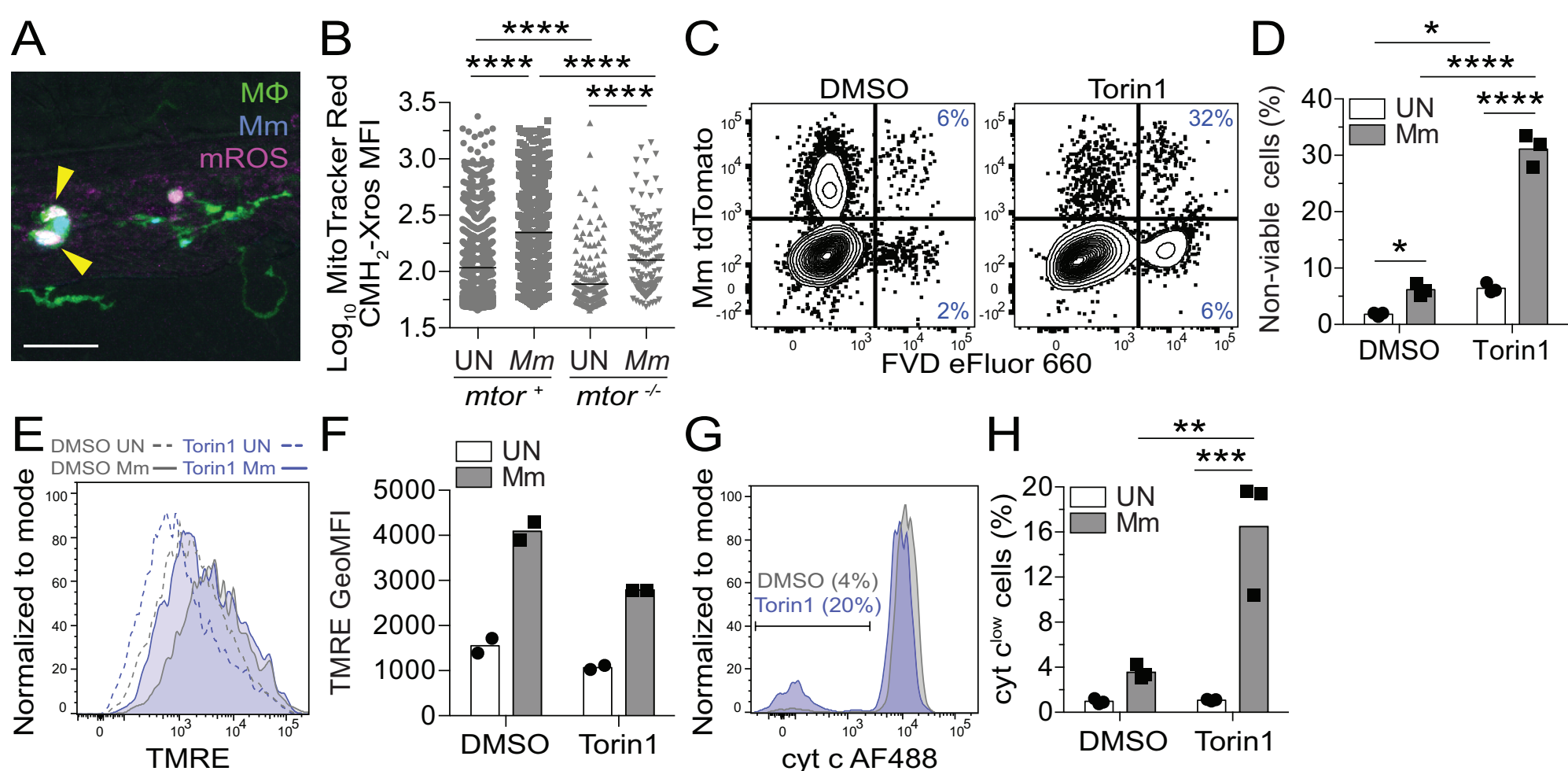
Figure 4

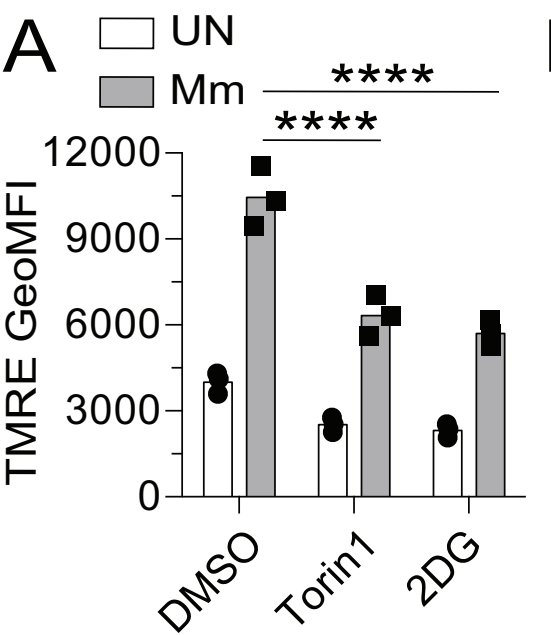

D

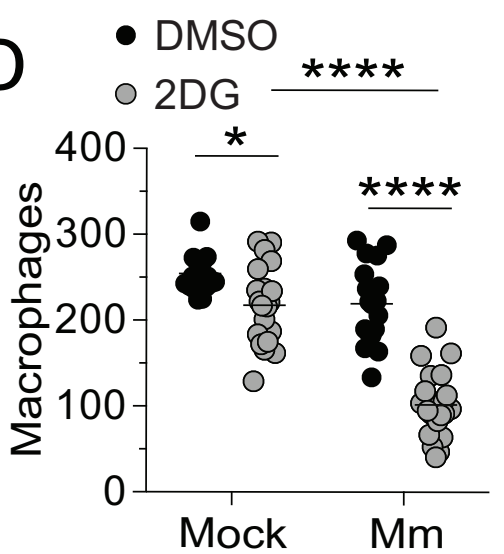

G

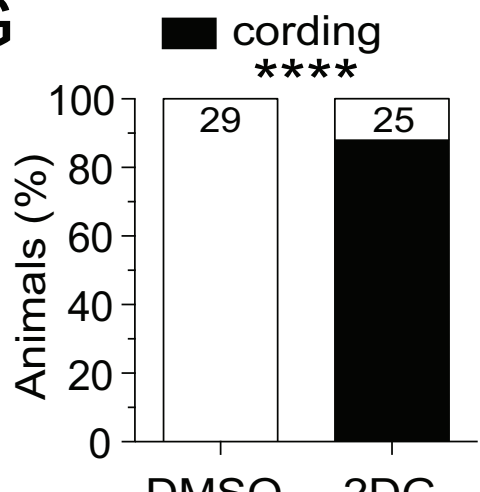

$\square$ not cording $\mathrm{H}$
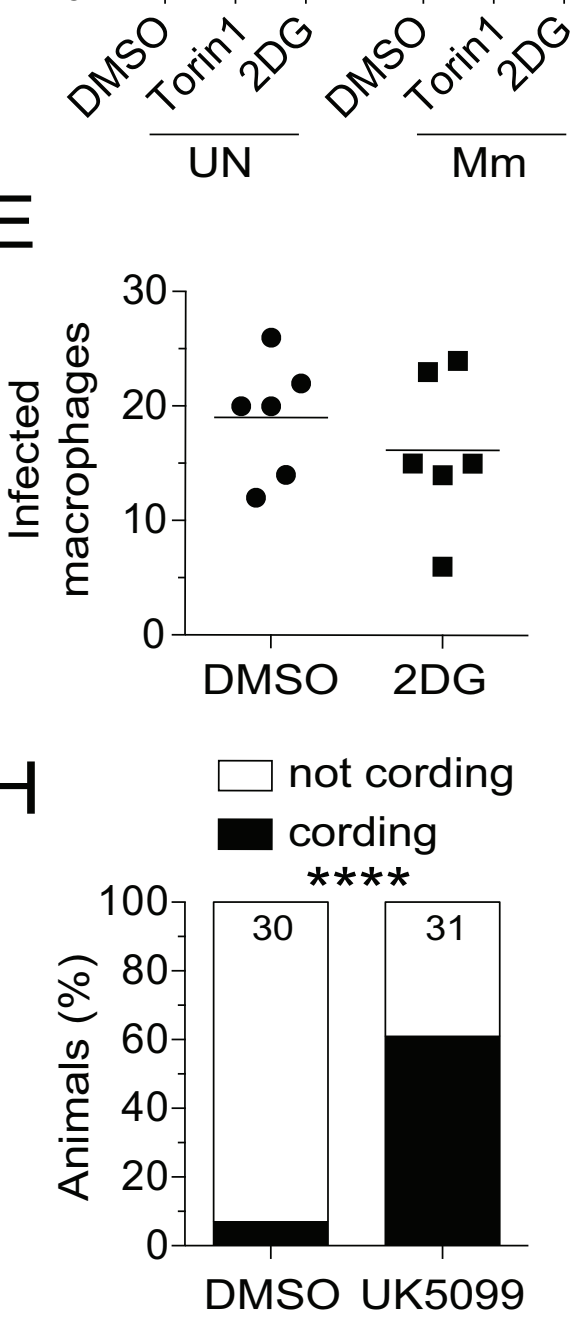

E
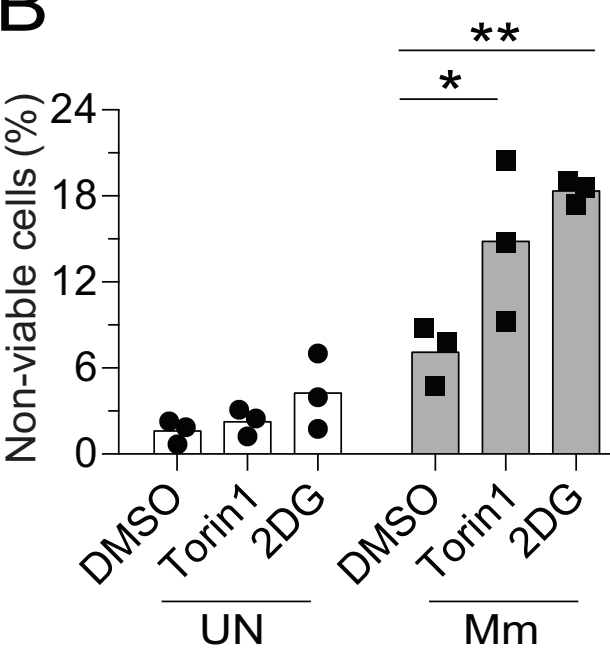

ว 1

0

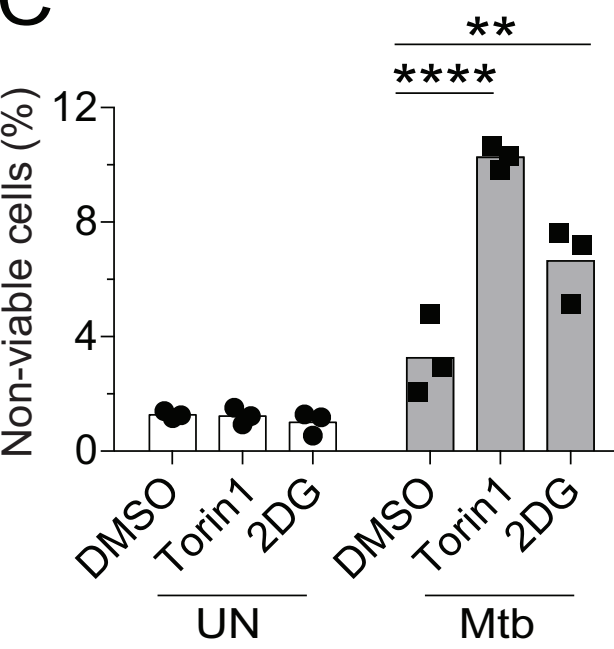

F
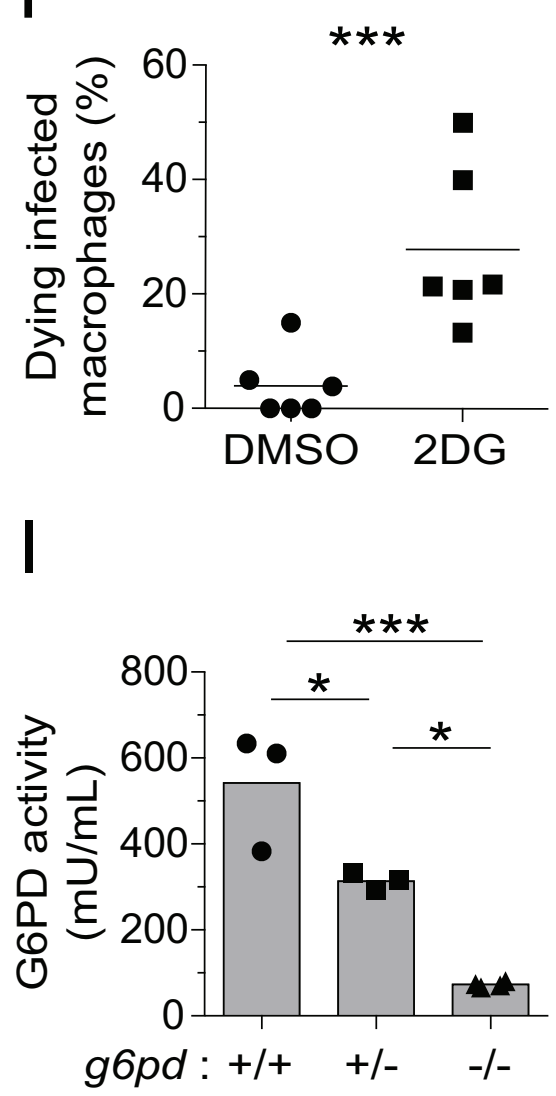

J

$\square$ not cording

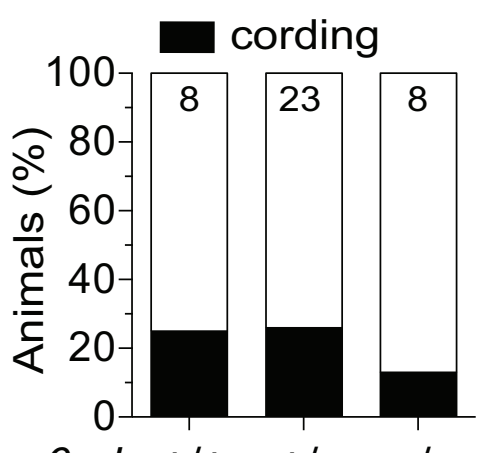

$\mathrm{K}$ g6pd : +/+ +/- -/-

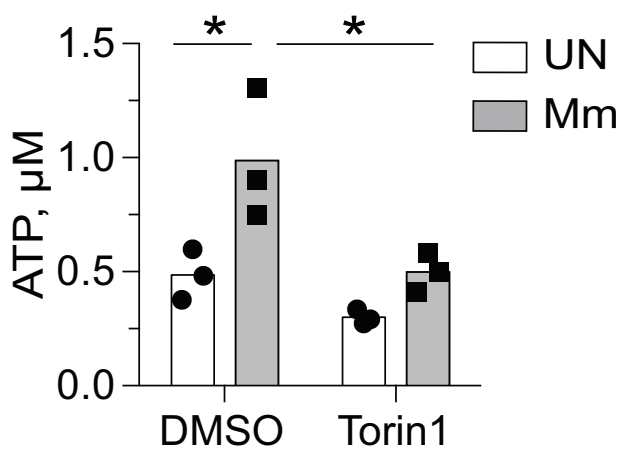



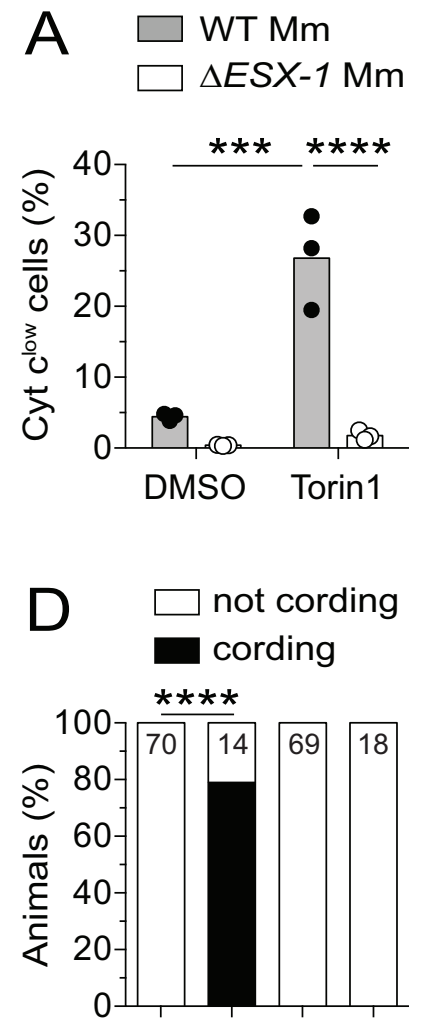

$$
\text { mtor: } \frac{+\quad-/}{\text { WT }} \frac{+-/-}{\Delta E S X-1}
$$

G

$\frac{0}{\frac{0}{0}}$

$$
n
$$
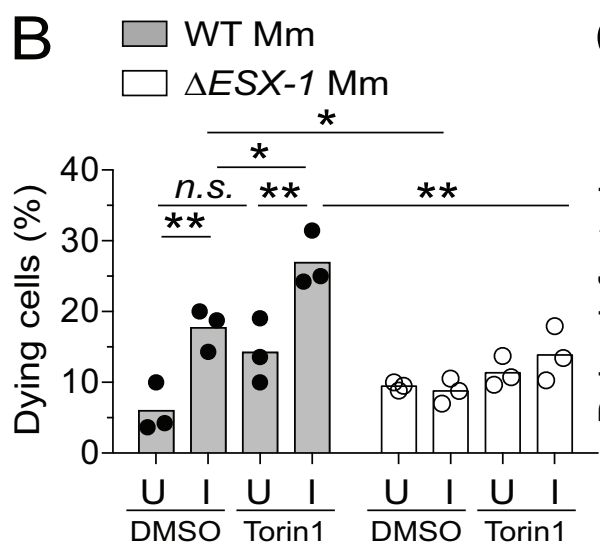

E
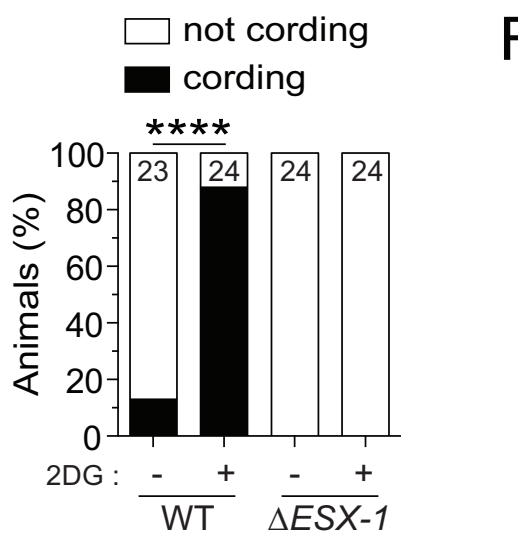

F
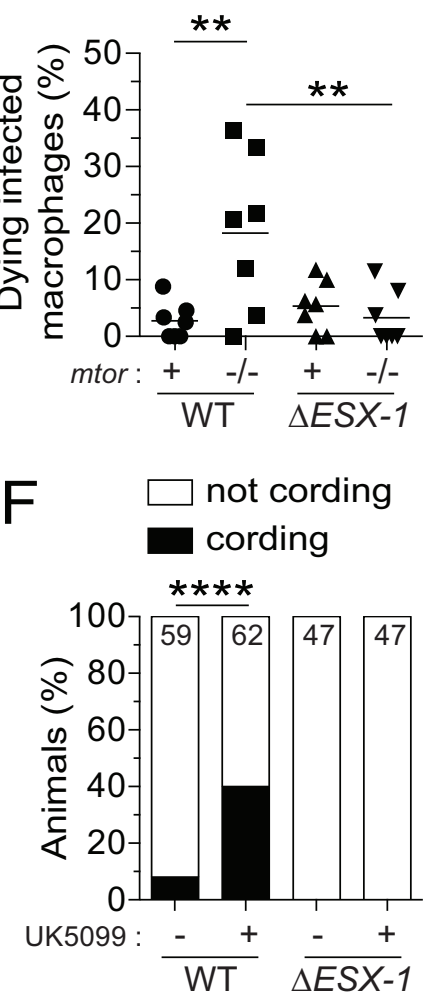

$* * *$
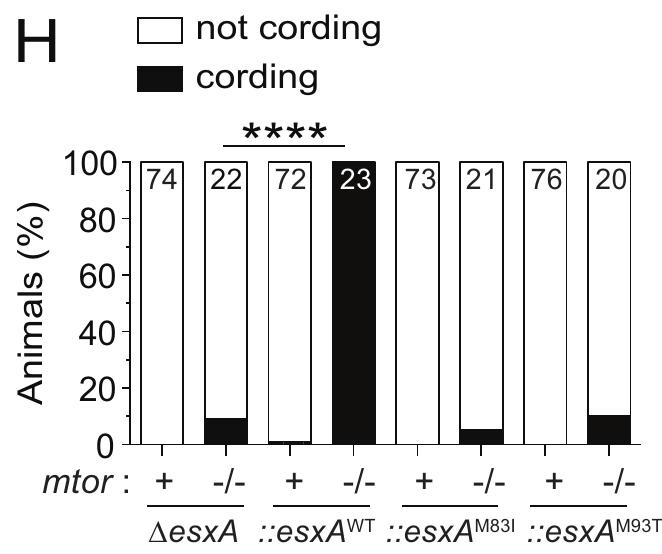


\section{Figure 6}
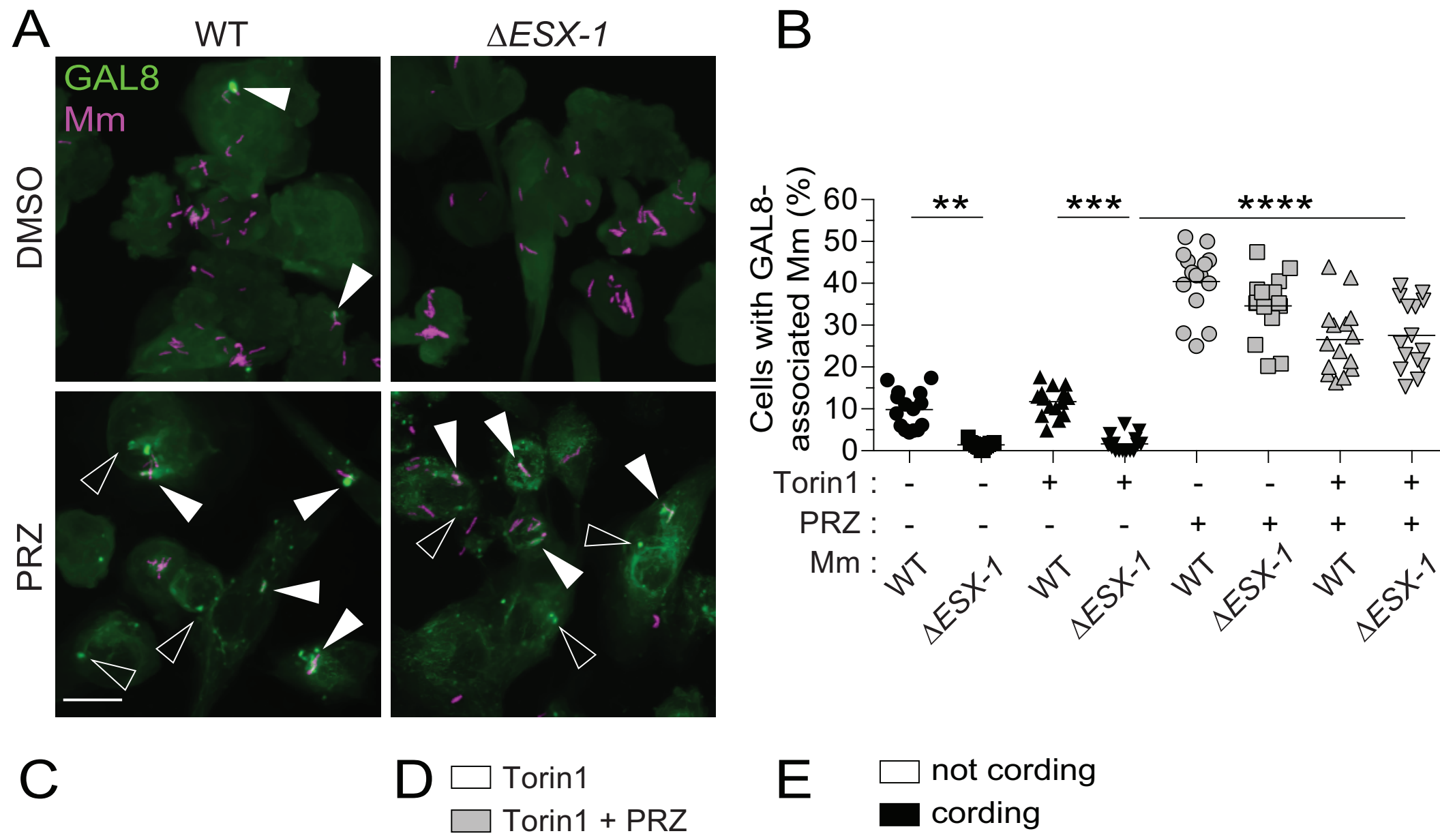

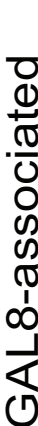

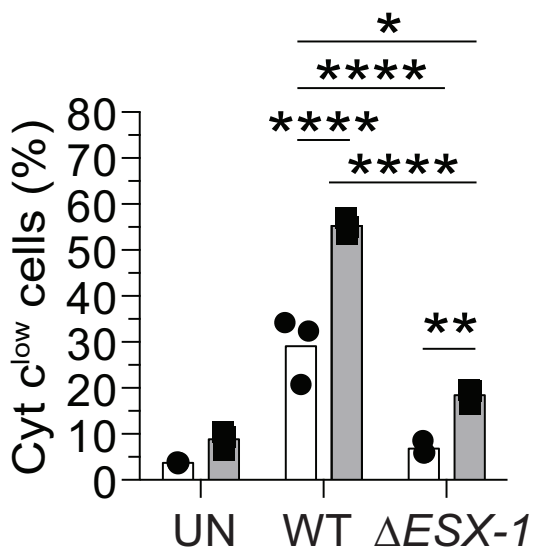

E $\square$ not cording
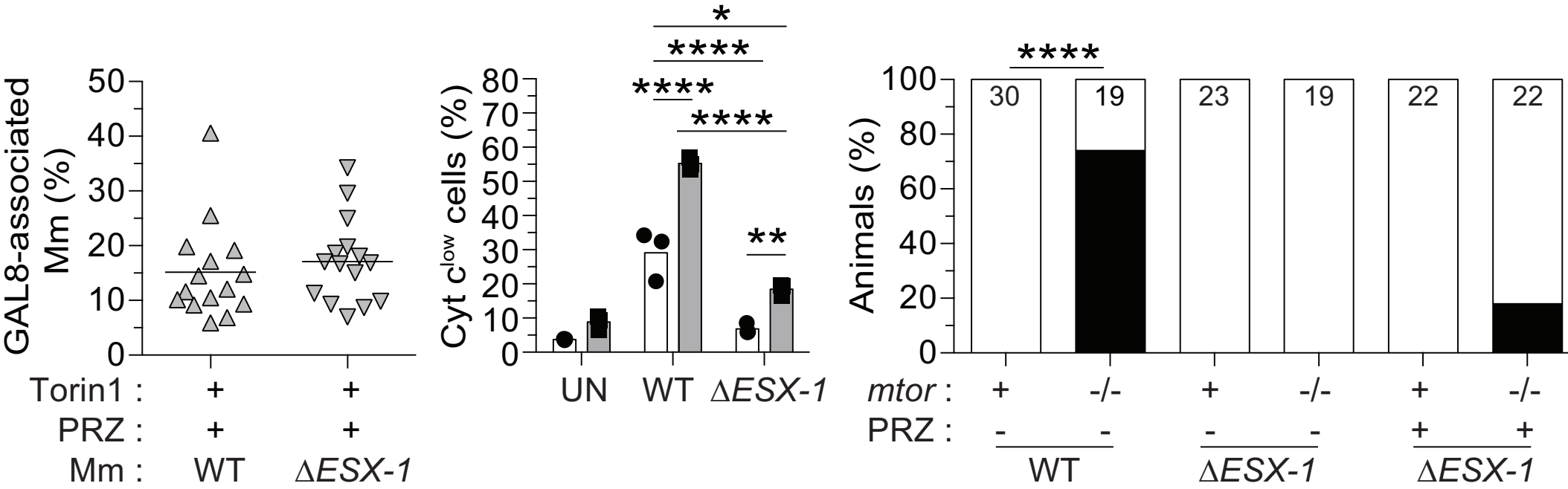
bioRxiv preprint doi: https://doi.org/10.1101/2022.01.30.478369; this version posted January 30,2022. The copyright holder for this preprint (which was not certified by peer review) is the author/funder, who has granted bioRxiv a license to display the preprint in perpetuity. It is made available under aCC-BY 4.0 International license.

\section{Figure 7}

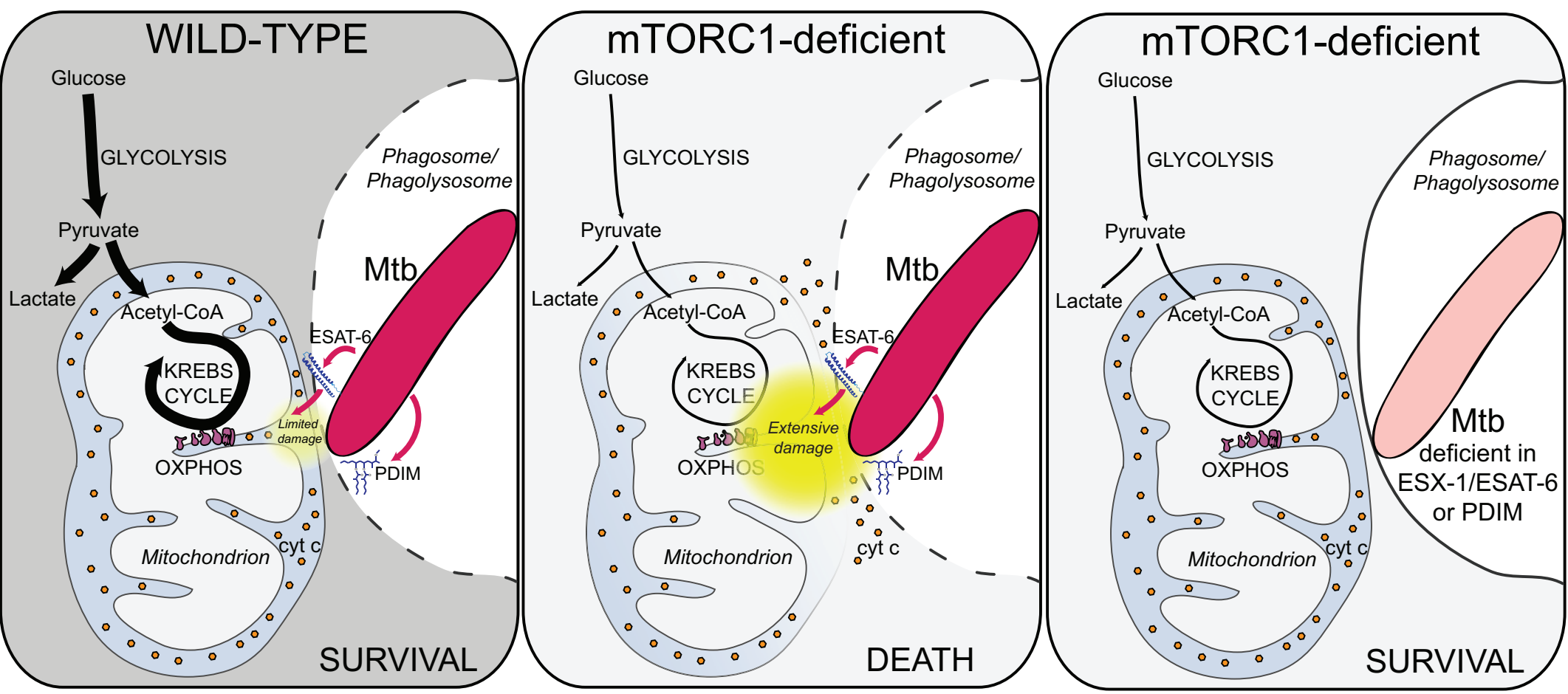

